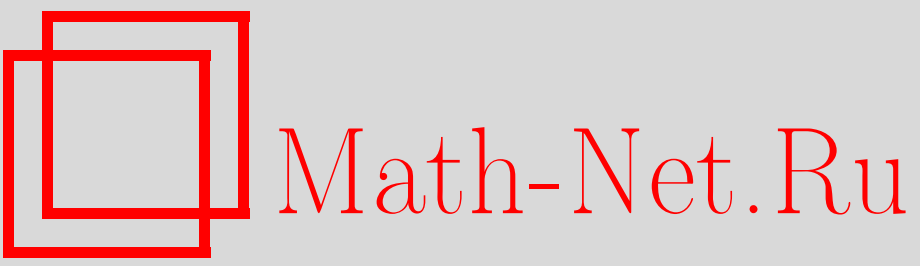

А. И. Комеч, Аттракторы нелинейных гамильтоновых одномерных волновых уравнений, УМH, 2000, том 55, выпуск 1, 45-98

DOI: https://doi.org/10.4213/rm249

Использование Общероссийского математического портала Math-Net.Ru подразумевает, что вы прочитали и согласны с пользовательским соглашением

http://www.mathnet.ru/rus/agreement

Параметры загрузки:

IP : 3.81 .55 .215

26 апреля 2023 г., 15:48:04 


\title{
АТТРАКТОРЫ НЕЛИНЕЙНЫХ ГАМИЛЬТОНОВЫХ ОДНОМЕРНЫХ ВОЛНОВЫХ УРАВНЕНИЙ
}

\begin{abstract}
А. И. Комеч
Строится теория аттракторов всех решений конечной энергии консервативных одномерных волновых уравнений на всей прямой. Для "невырожденных" уравнений (т.е. для уравнений “общего положения") аттрактором является множество всех стационарных решений. Любое решение конечной энергии сходится к этому аттрактору при $t \rightarrow \pm \infty$ в топологии Фреше, определяемой локальными энергетическими полунормами. Причиной такого притяжения является рассеяние энергии на бесконечности. Полученные результаты дают математическую модель боровских переходов между стационарными состояниями ("квантовых скачков") в квантовых системах.

Библиография: 67 названий.
\end{abstract}

\section{СОДЕРЖАНИЕ}

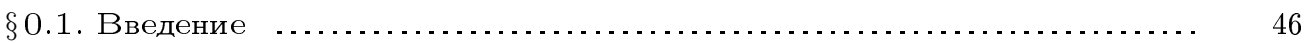

0.1.1. Боровские переходы к стационарным состояниям ............... 46

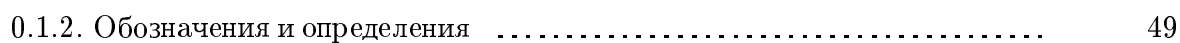

0.1.3. Рассеяние энергии в бесконечность .......................... 51

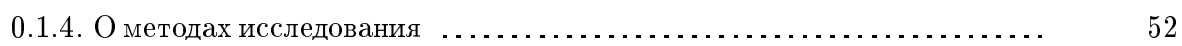

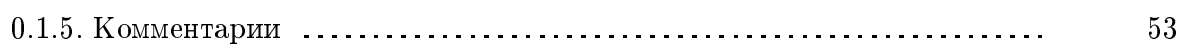

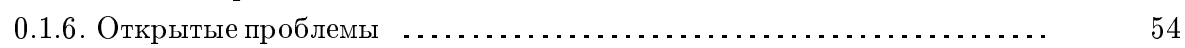

Глава І. Система Лэмба: струна с нелинейным осциллятором ......... 54

$\S 1.1$. Введение .............................................. 55

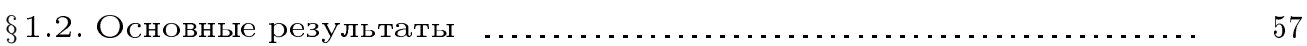

$\S 1.3$. Существование динамики и априорные оценки ................... 59

1.3.1. Существование динамики ................................. 59

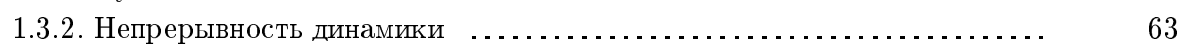

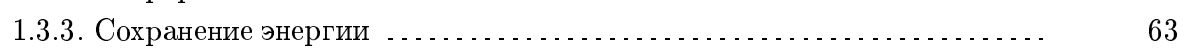

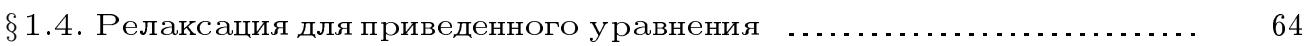

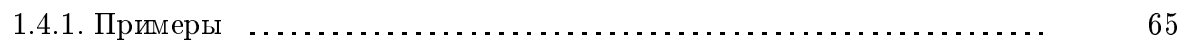

1.4.2. Доказательство релаксации для приведенного уравнения .......... 67

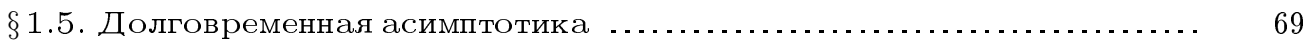

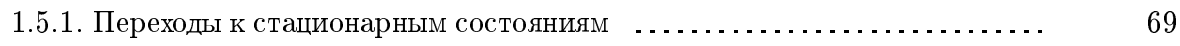

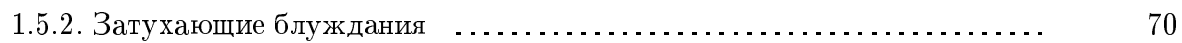

1.5.3. Транзитивность ............................................. 71

(С) А. И. Комеч 2000 
Глава II. Струна с конечным числом нелинейных осцилляторов . . . . . . . 71

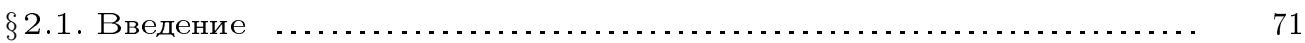

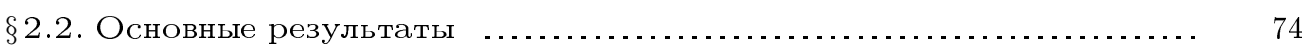

$\S 2.3$. Существование динамики и априорные оценки .................. 75

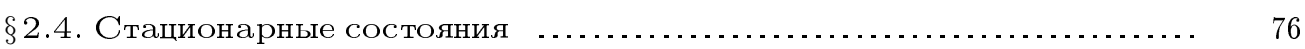

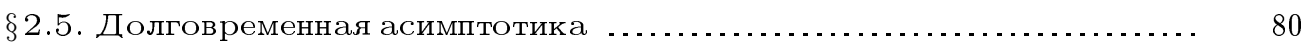

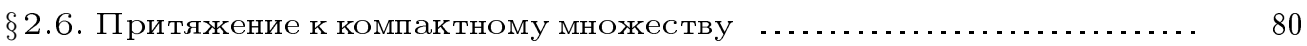

2.6.1. Релаксация на бесконечности ............................... 81

2.6.2. Рассеяние энергии в бесконечность … . . . . . . . . . . . . . . . . . . . 83

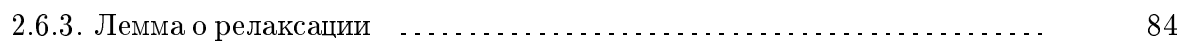

Глава III. Нелинейная струна с пространственно-локализованной нели-

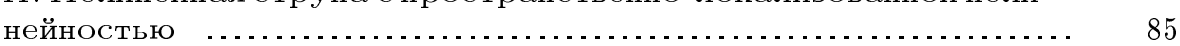

$\S 3.1$. Введение и основные результаты ............................. 85

$\S 3.2$. Существование динамики и априорные оценки .................. 86

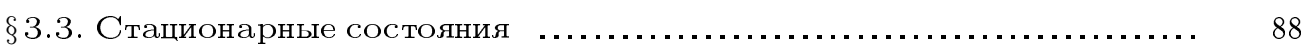

$\S 3.4$. Долговременная асимптотика ............................... 90

3.4.1. Компактное притягивающее множество ........................ 90

3.4.2. Доказательство теоремы 3.1 .3 ............................. 90

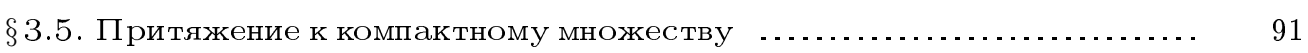

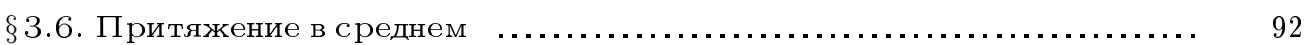

3.6.1. Рассеяние энергии в бесконечность .......................... 92

3.6.2. Нелинейная задача Гурса . . . . . . . . . . . . . . . . . . . . . . . . . . . 92

3.6.3. Доказательство притяжения в среднем ......................... 94

Список литературы . . . . . . . . . . . . .

\section{$\S$ 0.1. Введение}

Цель данной работы - исследование долговременной асимтотики всех решений конечной энергии нелинейных гамильтоновых волновых уравнений и систем.

0.1.1. Боровские переходы к стационарным состояниям. Вызывает удивление совершенно особая роль стационарных состояний во многих явлениях, описываемых гамильтоновыми нелинейными волновыми уравнениями. Перманентньй процесс воспроизводства стационарных состояний внушает мысль, что любое решение $Y(t)$ соответствующих уравнений стремится к некоторому (своему) стационарному пределу при $t \rightarrow \infty$ :

$$
Y(t) \rightarrow S_{ \pm}, \quad t \rightarrow \pm \infty
$$

Для краткости назовем такое поведение системы стабилизацией. Оно означает, что множество $\mathscr{S}$ всех стационарных состояний является точечным аттрактором соответствуюшего уравнения:

$$
Y(t) \rightarrow \mathscr{S}, \quad t \rightarrow \pm \infty
$$

Работа выполнена при частичной поддержке исследовательских грантов ИНТАС IR-97-0812 и РФФИ-ННИО 99-01-04012. 
Проблематика такого сорта сушествует давно в самых разных областях. Например, в классической электродинамике это проблема радиационного трения [23], [15], в квантовой теории это проблемы боровских переходов между стационарными состояниями [4], корпускулярно-волновой двойственности Л. де Бройля, устойчивости и классификации элементарных частиц. Кроме того, Э. Шрёдингер считал, что только квантовые переходы могут объяснить парадокс репродукции генов [52].

Переходы типа (0.1.1) постулируется в квантовой физике как экспериментальньй факт. Однако до сих пор не доказано, что такие переходы являются следствием динамических уравнений. Такое доказательство позволило бы дать внутреннее определение квантовых стационарных состояний как точек аттрактора динамических уравнений. Основная проблема состоит в том, что сходимость (0.1.1) представляется весьма парадоксальной и несовместимой с обратимостью и консервативностью гамильтоновых уравнений. Кроме того, такая сходимость невозможна для линейных автономных уравнений.

Главное содержание данной работы состоит в доказательстве того, что такая сходимость в принципе является возможным и даже типичным свойством для нелинейных гамильтоновых волновых уравнений вида

$$
\ddot{u}(x, t)=u^{\prime \prime}(x, t)+f(x, u(x, t)), \quad x \in \mathbb{R} .
$$

Мы доказьваем сходимость (0.1.1), (0.1.2) в гл. І для нелинейности, сосредоточенной в одной точке струны, $f(x, u)=\delta(x) F(u)$ (система Лэмба), ив гл. II - для нелинейности, сосредоточенной в конечном числе точек, $f(x, u)=\sum \delta\left(x-x_{k}\right) F_{k}(u)$. В гл. III эти результаты получены для общих одномерных нелинейных волновых уравнений и систем с пространственно-локализованной нелинейностью:

$$
f(x, u)=0, \quad|x| \geqslant \text { const } .
$$

Одним из основных итогов данного исследования является выбор подходящей топологии, в которой сходимость (0.1.1), (0.1.2) является возможной. Например, сходимость (0.1.1) в энергетической метрике, вообше говоря, невозможна ввиду сохранения энергии. Мы доказываем сходимость (0.1.1), (0.1.2) в топологии Фреше, определяемой локальньми энергетическими полунормами. Эта топология представляется оптимальной для притяжения к аттрактору в гамильтоновых уравнениях.

Главньм результатом данного исследования является обнаружение причины притяжения $(0.1 .1),(0.1 .2)$. Этой причиной является рассеяние энергии на бесконечности. Первоначально это рассеяние энергии было открыто в линейной и нелинейной теории рассеяния [8], [17]-[19], [22], [26], [42]-[45], [53], [57], [61]-[64], (см. обзоры [49], $[58])$ в случае, когда аттрактор $\mathscr{S}$ состоит из одной точки 0. При этом $(0.1 .1)$ и $(0.1 .2)$ эквивалентны хорошо известному "убыванию локальной энергии".

Линеаризация нелинейных задач в сочетании с теорией возмушений является мошным средством изучения многих нелинейных задач. Однако задачи долговременного поведения не могут быть решены в рамках линеаризованного подхода, если траектория не остается близкой к некоторому известному решению. Именно так обстоит дело, например, в проблемах радиационного трения, боровских переходов и многих других 
задачах. Поэтому для исследования долговременного поведения нелинейных гамильтоновых уравнений мы развиваем методы нелинейной теории рассеяния без использования теории возмушений.

Мы ограничиваемся исследованием решений конечной энергии. Решения бесконечной энергии здесь не рассматриваются, и для них долговременная асимптотика решаюшим образом зависит от поведения начальных данных на бесконечности.

В настояшее время эти результаты обобшены на системы, описываюшие взаимодействие классической заряженной частицы в трехмерном пространстве со скалярным волновым полем [38], [36], [35] или с электромагнитным полем Максвелла [37] (см. обзор [34]).

Отметим, что притяжение к аттрактору, состоящему из стационарных состояний, - хорошо изученное свойство диссипативных систем [1], [21], [40], [51], [60]. Их сушественное отличие от гамильтоновых уравнений состоит в том, что для диссипативных систем сходимость к аттрактору имеет место в глобальной энергетической метрике, но лишш при $t \rightarrow+\infty$.

Наши результаты показьвают, что асимптотики (0.1.1), (0.1.2) являются типичными свойствами "невырожденных" гамильтоновых уравнений, т.е. уравнений “общего положения". С другой стороны, многие примеры и численные эксперименты показывают, что для некоторых "исключительных" классов уравнений долговременное поведение решений может быть совершенно иным. Например, $G$-инвариантные уравнения с некоторой группой Ли симметрий $G$ (см. [20]) являются “исключительными" среди всех уравнений, и асимптотики (0.1.1), (0.1.2) в общем случае не имеют места для таких $G$-инвариантных уравнений. Действительно, для таких уравнений, вообще говоря, сушествуют “уединенные волны” [20], т.е. решения вида $\exp (i \Omega t) \Psi(x)$, где $\Omega$ - элемент соответствуюшей алгебры Ли. Асимптотика (0.1.1), вообще говоря, не вьполняется для такой уединенной волны, если $\Omega \neq 0$. Нашп результаты по стабилизации (0.1.1) соответствуют тривиальной группе симметрий $G=\{e\}$.

Существование нетривиальных уединенных волн для скалярных $U(1)$-инвариантных нелинейных гамильтоновых волновых уравнений доказано в [3]. Для $U(1)$-инвариантных нелинейных гамильтоновых систем Дирака, Максвелла-Дирака и Клейна-Гордона-Дирака такой результат получен в [13], [14].

В работах [5]-[7], [48], [55], [56] для ряда “невырожденных" $U(1)$-инвариантных систем доказано, что уединенные волны образуют аттрактор в топологии Фреше, но для системы Максвелла-Дирака это открытая проблема.

ЗАмЕчАниЕ. Система Янга-Миллса сильного взаимодействия инвариантна относительно группы симметрий $S U(2)$ [67] или $S U(3)$ [16], система электро-слабого взаимодействия инвариантна относительно группы симметрий $S U(2) \times U(1)$ [65]. Наши результаты для тривиальной группы симметрий $G=\{e\}$ и результаты [5]-[7], [48], [55], $[56]$ для $G=U(1)$ наводят на мысль о том, что аттрактор "невырожденных" $G$-инвариантных гамильтоновых уравнений состоит из “уединенных волн” $\exp (i \Omega t) \Psi(x)$. Эта гипотеза подтверждается также известными экспериментальными результатами о связи классификации элементарных частиц с классификацией алгебр Ли групп симметрий соответствуюших уравнений [16], [46]. 
0.1.2. Обозначения и определения. Мы рассматриваем некоторые классы одномерных уравнений вида

$$
\left\{\begin{array}{l}
\ddot{u}(x, t)=u^{\prime \prime}(x, t)+f(x, u(x, t)), \quad x \in \mathbb{R}, \\
\left.u\right|_{t=0}=u^{0}(x),\left.\quad \dot{u}\right|_{t=0}=v^{0}(x) .
\end{array}\right.
$$

Все производные здесь и ниже понимаются в смысле распределений, $u \in \mathbb{R}^{d}, d \geqslant 1$, и

$$
f(x, u)=-\nabla_{u} V(x, u),
$$

где $V(x, u)$ некоторая вешественная функция, назьваемая потенциалом. Тогда (0.1.5) является формально гамильтоновой системой с гамильтонианом

$$
\mathscr{H}(u, \dot{u})=\int\left(\frac{|\dot{u}|^{2}}{2}+\frac{\left|u^{\prime}\right|^{2}}{2}+V(x, u)\right) d x .
$$

Наши результаты касаются одномерных уравнений $(0.1 .5)$ с пространственно-локализованной нелинейностью, см. (0.1.4). Обозначим $Y(t)=(u(\cdot, t), \dot{u}(\cdot, t)), Y^{0}=$ $\left(u^{0}, v^{0}\right)$ и запишем задачу $(0.1 .5)$ в виде

$$
\dot{Y}(t)=\mathscr{V}(Y(t)), \quad t \in \mathbb{R}, \quad Y(0)=Y^{0} .
$$

Введем фазовое пространство состояний кqонечной энергии для задачи (0.1.8). Через $|\cdot|$ обозначим норму в $L^{2}\left(\mathbb{R}, \mathbb{R}^{d}\right)$ и через $|\cdot|_{R}, R>0,-$ норму в $L^{2}\left(B_{R}, \mathbb{R}^{d}\right)$, где $B_{R}-$ отрезок $[-R, R]$.

ОПРЕДЕЛЕНИЕ 0.1.1. i) $\mathscr{E}$-гильбертово пространство пар $(u(x), v(x)) \in C\left(\mathbb{R}, \mathbb{R}^{d}\right)$ $\oplus L^{2}\left(\mathbb{R}, \mathbb{R}^{d}\right)$ с конечной нормой

$$
\|(u, v)\|_{\mathscr{E}}=\left|u^{\prime}\right|+|u(0)|+|v| .
$$

ii) $\mathscr{E}_{F}$ - пространство $\mathscr{E}$ с топологией, определяемой локальными энергетическими полунормами

$$
\|(u, v)\|_{R}=\left\|u^{\prime}\right\|_{R}+\left|u\left\|_{R}+\right\| v\right|_{R}, \quad R>0 .
$$

Через $\stackrel{\mathscr{E}_{F}}{\longrightarrow}$ обозначается сходимость в этом пространстве.

ЗАМЕчАНИЕ. $\mathscr{E}_{F}$ - линейное топологическое счетно-нормированное пространство. Поэтому топология пространства $\mathscr{E}_{F}$ метризуема. Это означает, что сходимость $Y_{k} \stackrel{\mathscr{E}_{F}}{\longrightarrow} Y$ эквивалентна сходимости в некоторой метрике $\rho(\cdot, \cdot)$ в пространстве $\mathscr{E}_{F}$ (такая метрика, разумеется, не единственна). Кроме того, пространство $\mathscr{E}_{F}$ является сепарабельным.

Для краткости мы называем сепарабельные метризуемые пространства пространствами Фреше, даже если они не являются полными. В частности, $\mathscr{E}_{F}$ яВляется таким пространством Фреше. Как известно, в таких пространствах компактность подмножества эквивалентна его секвенциальной компактности.

Отметим, что гамильтониан $\mathscr{H}$ непрерывен на $\mathscr{E}$ и не является непрерывным в топологии Фреше пространства $\mathscr{E}_{F}$ во всех задачах, рассматриваемых в данной статье. 
ОПРЕДЕЛЕНИЕ 0.1.2. $\mathscr{S}=\{S \in \mathscr{E}: \mathscr{V}(S)=0\}$ - множество стационарных состояний задачи (0.1.8).

В каждой главе мы рассматриваем некоторьй класс уравнений вида (0.1.5), налагая подходящие условия на нелинейньй член $f(x, u)$. Для любого начального состояния $Y^{0} \in \mathscr{E}$ мы устанавливаем сушествование и единственность решения $Y(t) \in$ $C(\mathbb{R}, \mathscr{E})$ и сохранение энергии

$$
\mathscr{H}(Y(t))=\text { const }, \quad t \in \mathbb{R} \text {. }
$$

Наши главные результаты означают долговременное поведение следующих двух типов:

РЕЛАКСАЦИЯ. Для любого начального состояния $Y_{0} \in \mathscr{E}$ орбита $O(Y)=\{Y(t)$ : $t \in \mathbb{R}\}$ предкомпактна в $\mathscr{E}_{F} u$

$$
Y(t) \stackrel{\mathscr{E}_{F}}{\longrightarrow} \mathscr{S} \quad \text { npu } \quad t \rightarrow \pm \infty .
$$

Это по определению означает, что для любой окрестности $\mathscr{O}(\mathscr{S})$ множсества $\mathscr{S}$ в $\mathscr{E}_{F}$ существует такое $T>0$, ито $Y(t) \in \mathscr{O}(\mathscr{S})$ nрu $t>T$.

СТАБИЛИЗАцИя. Существуют такие стационарные состояния $S_{ \pm} \in \mathscr{S}$, зависящие от решения $Y(t)$, что

$$
Y(t) \stackrel{\mathscr{E}_{F}}{\longrightarrow} S_{ \pm} \quad \text { npu } \quad t \rightarrow \pm \infty .
$$

ЗАмечАниЕ. Пусть $\rho(\cdot, \cdot)$ - любая метрика, задаюшая топологию Фреше пространства $\mathscr{E}_{F}$. Тогда сходимость $(0.1 .11)$ эквивалентна сходимости в этой метрике

$$
\inf _{S \in \mathscr{S}} \rho(Y(t), S) \rightarrow 0 \text { при } t \rightarrow \pm \infty, \quad \forall R>0
$$

ввиду предкомпактности орбиты $O(Y)$. Это эквивалентно также сходимости в каждой полунорме

$$
\inf _{S \in \mathscr{S}}\|Y(t)-S\|_{R} \rightarrow 0 \text { при } t \rightarrow \pm \infty, \quad \forall R>0 .
$$

Из (0.1.11) вытекает (0.1.12), если множество $\mathscr{S}$ конечно. Импликацию $(0.1 .11) \Longrightarrow$ (0.1.12) можно формализовать при помощи следующего определения. Пусть $\mathscr{T}$ - подмножество некоторого топологического пространства $\mathscr{F}$.

ОПРЕДЕЛЕНИЕ 0.1.3. $\mathscr{T}$ - захватываюшее подмножество в $\mathscr{F}$, если из сходимости $Y(t) \stackrel{\mathscr{F}}{\longrightarrow} \mathscr{T}$ при $t \rightarrow \infty$ вытекает сходимость $Y(t) \stackrel{\mathscr{F}}{\longrightarrow} T$ при $t \rightarrow \infty$ к некоторой точке $T \in \mathscr{T}$ для каждой непрерьвной кривой $Y(t) \in C(\mathbb{R}, \mathscr{F})$ с предкомпактной в $\mathscr{F}$ орбитой $O(Y)$.

Например, замкнутое подмножество $Z \subset \mathbb{R}$ является захватывающим в $\mathbb{R}$ тогда и только тогда, когда

$$
Z \text { не содержит никакого непустого интервала }\left(c_{1}, c_{2}\right) \text {, }
$$

что эквивалентно также плотности $\mathbb{R} \backslash Z$ в $\mathbb{R}$. В частности, любое канторовское множество в $\mathbb{R}$ является захватьвающим подмножеством в $\mathbb{R}$. 
0.1.3. Рассеяние энергии в бесконечность. В настоящее время нет достаточно общего представления о типичном характере долговременного поведения решений нелинейных волновых уравнений. Не существует также и универсального метода анализа такого долговременного поведения. Поэтому мы изучаем здесь модельные задачи, допускающие исследование возможных типов долговременного поведения. Мы рассматриваем три типа таких задач M1-M3 и представляем результаты в соответствуюших главах.

Опишем кратко основные результаты. Мы устанавливаем долговременные асимптотики $(0.1 .11),(0.1 .12)$ для следуюших трех типов М1-М3 одномерных волновых уравнений (0.1.5).

M1. В гл. I рассматривается сингулярный нелинейный член вида $f(x, u)=$ $\delta(x) F(u)$, сосредоточенньй в одной точке $x=0$. Предполагается, что $d \geqslant 1$ и

$$
F(u) \in C^{1}\left(\mathbb{R}^{d}, \mathbb{R}^{d}\right), \quad F(u)=-\nabla V(u) \text { и } V(u) \rightarrow+\infty \text { при }|u| \rightarrow \infty .
$$

Тогда (0.1.11) верно для всех решений $Y(t) \in C(\mathbb{R}, \mathscr{E})$. Если, дополнительно, $Z=$ $\left\{z \in \mathbb{R}^{d}: F(z)=0\right\}$ - захватываюшее подмножество в $\mathbb{R}^{d}$, то выполняется также и (0.1.12). Для любых двух стационарных состояний $S_{ \pm} \in \mathscr{S}$ существуют решения $Y(t) \in C(\mathbb{R}, \mathscr{E})$, для которых вьполняется (0.1.12).

M2. В гл. II рассматривается сингулярный нелинейный член вида

$$
f(x, u)=\sum_{k=1}^{N} \delta\left(x-x_{k}\right) F_{k}(u),
$$

сосредоточенный в конечном множестве точек. Предполагается, что $d \geqslant 1$ и

$$
\begin{array}{ll}
\text { все } F_{k} \in C^{1}\left(\mathbb{R}^{d}, \mathbb{R}^{d}\right), & F_{k}(u)=-\nabla V_{k}(u), \\
\inf _{u \in \mathbb{R}^{d}} \min _{k} V_{k}(u)>-\infty \text { и } \max _{k} V_{k}(u) \rightarrow+\infty \text { при }|u| \rightarrow \infty .
\end{array}
$$

Тогда (0.1.11) имеет место для всех решений $Y(t) \in C(\mathbb{R}, \mathscr{E})$. Если, дополнительно, $d=1$ и все $F_{k}$ - действительные аналитические функции на $\mathbb{R}$, то и (0.1.12) также верно.

М3. В гл. III рассматриваются непрерывные нелинейные члены $f(x, u)$, для котоpыx $f(x, u)=0$ при $|x|>a$ для некоторого $a>0$. Предполагается, что $d \geqslant 1$ и

$$
\begin{aligned}
& f(x, u)=\chi(x) F(u), \quad F \in C^{1}\left(\mathbb{R}^{d}, \mathbb{R}^{d}\right), \quad \chi(x) \in C(\mathbb{R}), \\
& F(u)=-\nabla V(u) \text { и } V(u) \rightarrow+\infty \text { при }|u| \rightarrow \infty, \\
& \chi(x) \geqslant 0, \quad \chi(x) \not \equiv 0 \text { и } \chi(x)=0 \text { при }|x| \geqslant a .
\end{aligned}
$$

Тогда (0.1.11) вьполняется для всех решений $Y(t) \in C(\mathbb{R}, \mathscr{E})$. Если, дополнительно, $d=1$ и $F$ является действительной аналитической функцией на $\mathbb{R}$, то верно также и (0.1.12). 
ЗАмечАния. і) Система М1 введена Г. Лэмбом в [41] и рассматривалась также B [24].

ii) Система M1 является частным случаем M2, а M2 формально есть частный случай Мз. Однако мы рассматриваем все системы независимо по следующим причинам. Во-первых, мы рассматриваем модифицированную модель М1 и выводим для M1 (M2) более точные результаты, чем для M2 (M3). Во-вторых, методы, используемые для анализа системы М2 (M3), являются естественным развитием методов анализа системы M1 (M2).

0.1.4. О методах исследования. Все результаты статьи основаны на изучении рассеяния энергии в бесконечность. Энергия, рассеянная в бесконечность, может быть оценена снизу в каждой рассматриваемой задаче с помощью соответствующего “интеграла рассеяния". С другой стороны, эта энергия ограничена априори. Это приводит к оценке для интеграла рассеяния, обеспечивающей сходимость (0.1.11).

Мы развиваем методы глобального анализа задач с диссипацией $[1],[21],[40],[51]$, [60] для рассматриваемых гамильтоновых систем и применяем следующую общую схему.

I. Ограниченность интеграла рассеяния.

II. Убывание производных решения по времени.

III. Интегральное представление решения.

IV. Притяжение траектории в $\mathscr{E}_{F}$ к компактному (в $\mathscr{E}_{F}$ ) множеству.

V. Любая $\omega$-предельная (в $\mathscr{E}_{F}$ ) точка траектории является стационарным состоянием.

VI. $\mathscr{S}$ является захватьвающим подмножеством в $\mathscr{E}_{F}$.

Однако реализация этой схемы в рассматриваемых системах различна. Шаг IV для системы M2 вьводится при помощи понятия "релаксации на бесконечности" функции $f(t), t>0$, которое является ослабленной формой сходимости $f^{\prime}(t) \rightarrow 0$ при $t \rightarrow \infty$. Грубо говоря, оно означает, что $\max _{0 \leqslant \tau \leqslant T}|f(t+\tau)-f(t)| \rightarrow 0$ при $t \rightarrow \infty$ для каждого $T>0$. Это понятие обладает рядом удобных свойств (свойства R0-R8 из раздела 2.6.1), систематическое использование которых приводит к результату.

Для системы M3 шаг IV вытекает из исследования задачи Гурса для нелинейного волнового уравнения. При этом приближение к компакту $\mathscr{A}$ сначала удается получить лишш "в среднем":

$$
\int_{0}^{\infty} \rho_{R}^{2}(t) d t<\infty
$$

где $\rho_{R}(t)=\inf _{S \in \mathscr{A}}\|Y(t)-S\|_{R}$. Отсюда выводится, что на самом деле $\rho_{R}(t) \rightarrow 0$ при $t \rightarrow \pm \infty$. Для этого используется компактность $\mathscr{A}$, равномерная непрерьвность динамики вблизи компакта и соображения, напоминающие леммы Бореля-Кантелли.

C другой стороны, во всех системах M1-M3 шаг V вытекает из универсальной леммы 1.5.3 Ю. Е. Егорова [10], а шаг VI получается из следуюшего простого универсального критерия для $\mathscr{S} \subset \mathscr{E}_{F}$ быть захватывающим подмножеством.

Лемма 0.1.4. Пусть $\mathscr{F}_{1}$ и $\mathscr{F}_{2}$ - два пространства Фреше, отображение $I: \mathscr{F}_{1} \rightarrow \mathscr{F}_{2}$ непрерывно, $\mathscr{T}_{2}$ - захватывалющее подмножество в $\mathscr{F}_{2}$, I $\mathscr{T}_{1} \subset \mathscr{T}_{2}$ 
и I: $\mathscr{T}_{1} \rightarrow \mathscr{T}_{2}$ - инбективное отображение. Тогда $\mathscr{T}_{1}$ - захватывающее подмножество в $\mathscr{F}_{1}$.

В статье явно строится подобное отображение $I$ из $\mathscr{F}_{1}=\mathscr{E}_{F}$ в $\mathscr{F}_{2}=\mathbb{R}$ для всех систем M1-M3.

0.1.5. Комментарии. В статье устанавливается, что все решения конечной энергии рассматриваемых гамильтоновых систем сходятся при $t \rightarrow \pm \infty$ к аттрактору, состоящему возможно из бесконечного числа точек. Это обобшает результаты, известные для диссипативных систем [1], [21], [40], [51], [60]. Данное обобшение содержит следуюшие принципиальные отличия.

I. В диссипативных системах сходимость имеется, вообше говоря, лишь при $t \rightarrow+\infty$.

II. Причина этой сходимости совершенно другая. В диссипативных системах имеется поглощение энергии. В гамильтоновых же системах энергия сохраняется, а роль диссипации играет рассеяние энергии в бесконечность.

III. Такая сходимость, вообще говоря, отсутствует для гамильтоновых волновых уравнений в ограниченных областях из-за отражения волн от границы. Именно поэтому в статье уравнения (0.1.5) рассматриваются во всем пространстве.

IV. Для диссипативных систем сходимость (0.1.11) и (0.1.12) вьполняется в (глобальной) энергетической метрике соответствуюшего фазового пространства $\mathscr{E}$. Для гамильтоновых же систем сходимость (0.1.12) в метрике пространства $\mathscr{E}$, вообще говоря, невозможна ввиду сохранения энергии. Действительно, если $\left\|Y(t)-S_{ \pm}\right\|_{\mathscr{E}} \rightarrow 0$ при $t \rightarrow \pm \infty$, то из $(E)$ следует $\mathscr{H}\left(S_{ \pm}\right)=\mathscr{H}(Y(t))$, так как гамильтониан $\mathscr{H}$ непрерывен на $\mathscr{E}$ во всех рассматриваемых нами задачах. Поэтому сходимость в $\mathscr{E}$ всех решений конечной энергии означала бы, что $\mathscr{H}(\mathscr{E}) \subset \mathscr{H}(\mathscr{S})$. Однако это невозможно для любой нетривиальной гамильтоновой системы, если множество $\mathscr{S}$ дискретно. Аналогично, сходимость (0.1.12) всех решений невозможна для любой нетривиальной конечномерной гамильтоновой системы.

V. Для диссипативной системы динамическая группа состоит из компактных отображений $\mathscr{E}$, а для гамильтоновой - лишь из ограниченных, т.е. компактных в слабой топологии. Это отличие является главным, и оно представляет главную трудность в доказательстве асимптотик (0.1.11), (0.1.12). Именно оно определяет роль топологии Фреше в исследовании гамильтоновых систем.

Результаты гл. І показывают необходимость всех наложенных на систему M1 условий для справедливости основных результатов. Условия, налагаемые на систему М2, также близки к необходимьм, что иллюстрируется многочисленньми примерами в гл. II. То же относится и к условиям на систему М3 в гл. III.

Сходимость (0.1.12) означает "переход" вида

$$
S_{-} \mapsto S_{+}
$$

за время от $-\infty$ до $+\infty$. Это дает математическую модель боровских переходов между стационарными состояниями в квантовых системах. Для системы Лэмба M1 переходы (0.1.22) сушествуют для любых двух стационарных состояний $S_{ \pm} \in \mathscr{S}$ (леммa 1.2.4). 
Из сходимости (0.1.12) во всех рассматриваемых нами случаях вытекает неравенство

$$
\mathscr{H}\left(S_{ \pm}\right) \leqslant \mathscr{H}(Y(t)) \equiv \mathscr{H}\left(Y^{0}\right), \quad t \in \mathbb{R}
$$

подобно хорошо известному свойству слабой сходимости в гильбертовых и банаховых пространствах. Примеры показьвают, что строгое неравенство в (0.1.23) действительно возможно. Это можно назвать рассеянием энергии в бесконечность.

Результаты статьи показывают, что асимптотики (0.1.11) и (0.1.12) справедливы для "общих" систем и могут нарушаться в некоторых "исключительных" системах. А именно, сходимость (0.1.12) справедлива, если $\mathscr{S}$ - захватьваюшее подмножество, т.е. дискретное в некотором смысле. С другой стороны, многие примеры показывают, что для некоторых "узких" классов уравнений (0.1.5) долговременное поведение решений может быть совершенно отличным от (0.1.11) и (0.1.12). Например, это так для "вырожденных" систем М1 согласно лемме 1.2.3 и для $G$-инвариантных уравнений, рассмотренных в [20].

Главы I и II содержат обобшение результатов автора [27]-[32], результаты гл. III впервые опубликованы в [33].

0.1.6. Открытые проблемы. Сушествование стационарных состояний типа $(\Phi(x), \exp (i \omega t) \psi(x))$ доказано недавно в [13], [14] для нелинейных систем Дирака, Максвелла-Дирака и Клейна-Гордона-Дирака. Тем не менее, долговременная сходимость типа (0.1.11) или $(0.1 .12)$ к этим стационарным состояниям является открытой проблемой.

Долговременные асимптотики (0.1.11) и (0.1.12) еше не доказаны для релятивистки-инвариантных уравнений (0.1.3), т.е. в случае, когда нелинейньй член $f(x, u)=$ $F(u)$ не зависит от $x$. Если асимптотики (0.1.12) справедливы для решений такого уравнения, то для них также имеют место солитоно-подобные асимптотики. Это вытекает из релятивистской инвариантности уравнения.

Автор выражает глубокую благодарность Х. Брезису, М. И. Вишику, А.И. Шнирельману и Г. Шпону за постоянное внимание к работе и плодотворные дискуссии.

\section{Глава І. Система Лэмба: струна с нелинейньм осциллятором}

Сходимость к стационарным состояниям доказьвается для системы уравнений, описывающей взаимодействие бесконечной струны с нелинейным осциллятором. Эта система была рассмотрена Г. Лэмбом для случая линейного осциллятора [41]. Система формально эквивалентна одномерному волновому уравнению с нелинейным членом $\delta(x) F(u)$, сосредоточенньм в точке $x=0$. Предельные стационарные состояния, соответствуюшие $t= \pm \infty$, могут быть различными и произвольными. Задача сводится к исследованию приведенного обыкновенного нелинейного уравнения для осциллятора. Глава содержит обобшение результатов автора [27]-[30]. 


\section{$\S$ 1.1. Введение}

Мы устанавливаем сходимость типа (0.1.11), (0.1.12) к стационарным состояниям для решений $u(x, t)$ системы

$$
\begin{aligned}
& \ddot{u}(x, t)=u^{\prime \prime}(x, t), \quad x \in \mathbb{R} \backslash\{0\}, \\
& m \ddot{y}(t)=F(y(t))+u^{\prime}(0+, t)-u^{\prime}(0-, t), \quad y(t) \equiv u(0, t) .
\end{aligned}
$$

Решения $u(x, t)$ принимают значения в $\mathbb{R}^{d} \mathrm{c} d \geqslant 1$. Мы рассматриваем задачу Коши для системы (1.1) с начальньми условиями

$$
\left.u\right|_{t=0}=u^{0}(x) ;\left.\quad \dot{u}\right|_{t=0}=v^{0}(x) ;\left.\quad \dot{y}\right|_{t=0}=p^{0} .
$$

Последнее условие в (1.1.2) необходимо только в случае $m>0$. Ниже мы рассматриваем случай $m>0$ и иногда комментируем разницу со случаем $m=0$. Физически, задача (1.1.1) описывает малые поперечные колебания бесконечной струны, натянутой параллельно оси $0 x$; частица массы $m \geqslant 0$ закреплена на струне в точке $x=0$; $F(y)$ - внешнее (нелинейное) силовое поле, действуюшее на частищу, перпендикулярно к оси $0 x$ (рис. 1$)$.

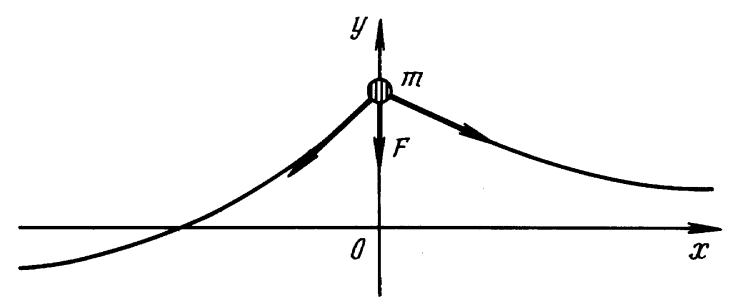

Рис. 1

Система (1.1.1) формально эквивалентна одномерному нелинейному волновому уравнению с нелинейньм членом $\delta(x) F(u)$, сосредоточенным в точке $x=0$ (и с массой $m$, сосредоточенной в той же точке):

$$
(1+m \delta(x)) \ddot{u}(x, t)=u^{\prime \prime}(x, t)+\delta(x) F(u(x, t)), \quad(x, t) \in \mathbb{R}^{2} .
$$

В линейном случае, когда $F(y)=-k y, k>0$, система (1.1.1) имеет единственное стационарное решение конечной энергии $s(x) \equiv 0$. В этом случае стабилизация к нулю рассмотрена впервые Г. Лэмбом [41].

Введем фазовое пространство состояний конечной энергии для системы (1.1.1) в случае $m>0$ (соответственно $m=0$ ). Обозначим через $L^{2}$ гильбертово пространство $L^{2}\left(\mathbb{R}, \mathbb{R}^{d}\right)$ с нормой $|\cdot|$. 
ОПРЕДЕЛЕНИЕ 1.1.1. і) $\mathscr{E}\left(\mathscr{E}^{0}\right)$ - гильбертово пространство троек $(u(x), v(x), p) \in$ $C\left(\mathbb{R}, \mathbb{R}^{d}\right) \oplus L^{2} \oplus \mathbb{R}^{d}$ (или пар $\left.(u(x), v(x)) \in C\left(\mathbb{R}, \mathbb{R}^{d}\right) \oplus L^{2}\right)$ с $u^{\prime}(x) \in L^{2}$ и нормой

$$
\begin{gathered}
\|(u, v, p)\|_{\mathscr{E}}=\left\|u^{\prime}\right\|+|u(0)|+\|v\|+|p| \\
\left(\|(u, v)\|_{\mathscr{E} 0}=\left\|u^{\prime}\right\|+|u(0)|+\|v\|\right) .
\end{gathered}
$$

ii) $\mathscr{E}_{F}-$ пространство $\mathscr{E}$ с топологией Фреше, определяемой полунормами

$$
\|(u, v, p)\|_{R} \equiv\left|u^{\prime}\right|_{R}+|u(0)|+\|v\|_{R}+|p|, \quad R>0
$$

где $|\cdot|_{R}$ - норма в $L^{2}\left(-R, R ; \mathbb{R}^{d}\right)$.

Мы предполагаем выполнение условий (0.1.15). Система (1.1.1) формально является гамильтоновой с фазовым пространством $\mathscr{E}$ и функцией Гамильтона

$$
\mathscr{H}(u, v, p)=\frac{1}{2} \int_{\mathbb{R}}\left[|v(x)|^{2}+\left|u^{\prime}(x)\right|^{2}\right] d x+m \frac{|p|^{2}}{2}+V(u(0))
$$

для $(u, v, p) \in \mathscr{E}$. Мы рассматриваем решения $u(x, t)$, для которых

$$
Y(t)=(u(\cdot, t), \dot{u}(\cdot, t), \dot{y}(t)) \in C(\mathbb{R}, \mathscr{E}), \quad y(t) \equiv u(0, t)
$$

и записываем задачу Коши (1.1.1), (1.1.2) в виде

$$
\dot{Y}(t)=\mathscr{V}(Y(t)) \text { при } t \in \mathbb{R}, \quad Y(0)=Y^{0},
$$

где $Y^{0}=\left(u^{0}, v^{0}, p^{0}\right)$. Обсудим постановку этой задачи Коши для функций $Y(t) \in$ $C(\mathbb{R}, \mathscr{E})$. Во-первых, $u \in C\left(\mathbb{R}^{2}, \mathbb{R}^{d}\right)$, так как $Y(t) \in C(\mathbb{R}, \mathscr{E})$. Поэтому первое уравнение в (1.1.1) эквивалентно разложению Даламбера

$$
u(x, t)=f_{ \pm}(x-t)+g_{ \pm}(x+t), \quad \pm x>0,
$$

где

$$
f_{ \pm}, g_{ \pm} \in C\left(\mathbb{R}, \mathbb{R}^{d}\right)
$$

Следовательно,

(1.1.10) $\dot{u}(x, t)=-f_{ \pm}^{\prime}(x-t)+g_{ \pm}^{\prime}(x+t), \quad u^{\prime}(x, t)=f_{ \pm}^{\prime}(x-t)+g_{ \pm}^{\prime}(x+t)$ при $\pm x>0$, где все производные понимаются в смысле обобшенных функций. Из предположения $Y(t) \in C(\mathbb{R}, \mathscr{E})$ вытекает, что

$$
f_{ \pm}^{\prime}, g_{ \pm}^{\prime} \in L_{\mathrm{loc}}^{2}\left(\mathbb{R}, \mathbb{R}^{d}\right)
$$

Обсудим смысл второго уравнения в (1.1.1). 
ОПРЕДЕЛЕНИЕ 1.1.2. Во втором уравнении (1.1.1) положим

$$
u^{\prime}(0 \pm, t) \equiv f_{ \pm}^{\prime}(-t)+g_{ \pm}^{\prime}(t) \in L_{\mathrm{loc}}^{2}\left(\mathbb{R}, \mathbb{R}^{d}\right)
$$

где производная $\ddot{y}(t)$ от функции $y(t) \equiv u(0 \pm, t) \in C\left(\mathbb{R}, \mathbb{R}^{d}\right)\left(\right.$ или от $\left.\dot{y}(t) \in L_{\mathrm{loc}}^{2}\left(\mathbb{R}, \mathbb{R}^{d}\right)\right)$ понимается в смысле обобшенных фукций.

Отметим, что функции $f_{ \pm}$и $g_{ \pm}$в (1.1.8) определены однозначно с точностью до прибавления константы. Поэтому определение (1.1.12) однозначно.

Стационарные состояния $S=(s(x), 0,0) \in \mathscr{E}$ для (1.1.7) легко находятся. Определим для каждого $c \in \mathbb{R}^{d}$ постоянную функцию

$$
s_{c}(x)=c \text { при } x \in \mathbb{R} .
$$

Тогда множество $\mathscr{S}$ всех стационарных состояний $S \in \mathscr{E}$ имеет вид

$$
\mathscr{S}=\left\{S_{z}=\left(s_{z}(x), 0,0\right): z \in Z\right\},
$$

где $Z=\left\{z \in \mathbb{R}^{d}: F(z)=0\right\}$. Назовем систему (1.1.1) и потенциал $V(u)$ "невырожденными", если множество $Z$ - захватывающее подмножество в $\mathbb{R}^{d}$. Для $d=1$ это означает, что справедливо условие (T1) (см. введение), что эквивалентно также условию

$$
F(u) \not \equiv 0 \text { на каждом непустом интервале } c_{1}<u<c_{2} .
$$

Главный результат данной главы означает, что множество $\mathscr{S}$ является точечным аттрактором системы (1.1.1) в топологии Фреше пространства $\mathscr{E}_{F}$.

\section{§ 1.2. Основные результаты}

Начнем с построения динамики.

ПРЕДЛОЖЕНИЕ 1.2.1. Пусть $m>0$ в системе (1.1.1), $d \geqslant 1$ и выполняется условие (0.1.15). Тогда справедливы следующие утверждения.

i) Для любого $Y^{0} \in \mathscr{E}$ задача Коши (1.1.7) имеет единственное решение $Y(t) \in C(\mathbb{R}, \mathscr{E})$.

ii) Отображсние $W_{t}: Y^{0} \mapsto Y(t)$ непрерывно в $\mathscr{E}$ и $\mathscr{E}_{F}$ при любом $t \in \mathbb{R}$.

iii) Выполняется закон сохранения әнергии $(E)$.

ЗАМЕЧАниЕ. В случае $m=0$ нужно отбросить последнее условие в (1.1.2). В этом случае предложение 1.2 .1 остается справедливьм с заменой $\mathscr{E}$ на $\mathscr{E} 0$.

Наш главный результат здесь - следуюшая теорема. 
ТЕОРема 1.2.2. Пусть выполняются условия предложения 1.2 .1 и начальное состояние $Y^{0} \in \mathscr{E}$. Тогда справедливы следующие утвержсдения.

i) Для решения $Y(t) \in C(\mathbb{R}, \mathscr{E})$ задачи Коши (1.1.7) орбита $O(Y)$ предкомпактна в $\mathscr{E}_{F}$ u

$$
Y(t) \stackrel{\mathscr{E}_{F}}{\longrightarrow} \mathscr{S} \quad \text { npu } \quad t \rightarrow \pm \infty
$$

ii) Пусть, дополнительно, множество $Z$ - захватывающее подмножество в $\mathbb{R}^{d}$. Тогда существуют стационарные состояния $S_{ \pm} \in \mathscr{S}$, зависящие от решения $Y(t)$, для которьх

$$
Y(t) \stackrel{\mathscr{E}_{F}}{\longrightarrow} S_{ \pm} \quad \text { npu } \quad t \rightarrow \pm \infty
$$

iii) Пусть $d=1$. Тогда (1.2.2) выполняется при любом множсестве $Z$, если

$$
u^{0}(x)=C_{ \pm}, \quad v^{0}(x)=0 \quad \partial \Omega я \quad \pm x>r^{0}
$$

при некоторых $r^{0} \geqslant 0$ и $C_{ \pm} \in \mathbb{R}$.

ЗАмечАния. і) Достаточно проверить (1.2.1) и (1.2.2) только для $t \rightarrow+\infty$, так как система (1.1.1) обратима.

ii) Из сходимости (1.2.2) и из (1.1.6), (0.1.15) по лемме Фату вытекает (0.1.23).

iii) Аналогичная теорема имеет место в случае $m=0$.

Сходимость (1.2.2) - это типичное свойство “невырожденных" систем с захватываюшим множеством $Z$. Возникает вопрос о возможно более сложном долговременном поведении решений “вырожденных” систем (1.1.1), когда множество стационарных состояний содержит некоторые непрерьвные компоненты. Тогда можно ожидать комбинацию приближения (1.2.1) к этим компонентам с “затухаюшими блужданиями" вдоль этих компонент. Следующая лемма описывает пример такого более сложного поведения. Она показьвает, что при $d=1$ для сходимости (1.2.2) всех решений $Y(t) \in C(\mathbb{R}, \mathscr{E})$ условие $(N D)$ является необходимым.

ЛЕмма 1.2.3. Пусть $d=1$ и все условия предложения 1.2 .1 выполнены, $а$ условие $(N D)$ нарушено. Тогда существуют решения $Y(t) \in C(\mathbb{R}, \mathscr{E})$ системь (1.1.7), для которых (1.2.2) не выполняется.

ЗАмечАния. і) Из теоремы 1.2.2 і) следует справедливость (1.2.1) для решений $Y(t)$ из леммы 1.2.3. Следовательно, эти решения описьвают затухающие блуждания вдоль аттрактора $\mathscr{S}$.

ii) Из теоремы 1.2.2 iii) вытекает, что (1.2.3) не вьполнено для начальных данных $Y(0)$ всех решений из леммы 1.2.3.

Далее возникает вопрос о связи между предельными стационарными состояниями $S_{ \pm}$решений системы (1.1.7) при $t \rightarrow \pm \infty$. Следующая лемма означает, что предельные стационарные состояния в (1.2.2) могут быть произвольньми. 
Лемма 1.2.4. Пусть $d \geqslant 1$ и $F(y) \in C\left(\mathbb{R}^{d}, \mathbb{R}^{d}\right)$ в системе (1.1.1). Тогда для любых двух стационарных состояний $S_{ \pm} \in \mathscr{S}$ существуют решения $Y(t) \in C(\mathbb{R}, \mathscr{E})$ системы (1.1.7), связывающие $S_{ \pm}$в смыслле (1.2.2).

ЗАмечАнИЕ. Лемма 1.2.4 означает, что в системе (1.1.1) нет принципа исключения. Иньми словами, это система с нетривиальными "боровскими" переходами между различньтии стационарными состояниями $S_{+} \neq S_{-}$. Такие переходы являются чисто нелинейным эффектом, который невозможен в линейных автономных уравнениях Шрёдингера или Дирака.

ПримеР. В качестве тривиального примера можно рассмотреть систему (1.1.1) с $d \geqslant 1$ в случае $m=0$ и $F(y) \equiv 0$. В этом случае система (1.1.1) совпадает с линейным уравнением Даламбера, и оба условия (0.1.15), $(N D)$ не вьполняются. Соответственно для решений $Y(t) \in C(\mathbb{R}, \mathscr{E})$, вообше говоря, орбита $O(Y)$ не является предкомпактом в $\mathscr{E}_{F}$, и сходимость $(1.2 .2)$ отсутствует. В то же время здесь имеется сходимость (1.2.1) и справедливы утверждения теоремы 1.2.2 iii) и лемм 1.2.3, 1.2.4. Это легко видно из формулы Даламбера для решения задачи Коши.

В $\S 1.3$ мы выводим приведенное уравнение для нелинейного осциллятора и доказьваем предложение 1.2.1. В $\S 1.4$ мы строим долговременную асимптотику решений приведенного уравнения и приводим типичные примеры.

В $\S 1.5$ доказывается теорема 1.2.2 и леммы 1.2.3, 1.2.4.

\section{§ 1.3. Существование динамики и априорные оценки}

Мы доказываем здесь предложение 1.2.1.

1.3.1. Существование динамики. Сначала докажем единственность решения $Y(t)=(u(\cdot, t), \dot{u}(\cdot, t), \dot{y}(t)) \in C(\mathbb{R}, \mathscr{E})$, предполагая его сушествование. При этом будет получен и алгоритм его построения, т.е. по сушеству будет доказано и его существование.

Единственность решения. Из начальных условий (1.1.2) и разложения Даламбера (1.1.8) вытекают хорошо известные формулы для $f_{ \pm}(z)$ и $g_{ \pm}(z)$ в области $\pm z>0$ :

$$
\begin{array}{ll}
f_{ \pm}(z)=\frac{u^{0}(z)}{2}-\frac{1}{2} \int_{0}^{z} v^{0}(y) d y+C_{ \pm}, & \pm z>0, \\
g_{ \pm}(z)=\frac{u^{0}(z)}{2}+\frac{1}{2} \int_{0}^{z} v^{0}(y) d y-C_{ \pm}, & \pm z>0,
\end{array}
$$

где $C_{ \pm}$- произвольные константы. Поскольку $f_{ \pm}$и $g_{ \pm}$в (1.1.8) определены с точностью до константы, то будем считать $C_{ \pm}=0$. Из (1.3.1) вытекает

$$
f_{ \pm}^{\prime}(z), g_{ \pm}^{\prime}(z) \in L^{2}\left(\mathbb{R}_{ \pm}, \mathbb{R}^{d}\right)
$$

так как $\left(u^{0}, v^{0}, p^{0}\right) \in \mathscr{E}$, где $\mathbb{R}_{ \pm} \equiv\{x \in \mathbb{R}: \pm x>0\}$. Из (1.3.1) следует справедливость обычной формулы Даламбера для $|x| \geqslant|t|$ :

$$
u(x, t)=\frac{u^{0}(x-t)+u^{0}(x+t)}{2}+\frac{1}{2} \int_{x-t}^{x+t} v^{0}(y) d y .
$$


Таким образом, решение $u(x, t)$ в области $|x| \geqslant|t|$ определено однозначно. Остается доказать единственность решения в области $|x|<|t|$.

Рассмотрим случай $t>0$. Тогда в области $|x|<t$ в разложении (1.1.8) неизвестными функциями являются $f_{+}(x-t)$ при $0<x<t$ и $g_{-}(x+t)$ при $-t<x<0$. Итак, нужно найти функции $f_{+}(z)$ при $z<0$ и $g_{-}(z)$ при $z>0$. Для нахождения этих неизвестных функций выведем обыкновенное нелинейное дифференциальное уравнение для $y(t) \equiv u(0, t)$.

Лемма 1.3.1. Для любого решения $Y(t)=(u(\cdot, t), \dot{u}(\cdot, t), \dot{y}(t)) \in C(\mathbb{R}, \mathscr{E})$ системы (1.1.7) функиия $y(t) \equiv u(0, t)$ является решением “приведенного” уравнения

$$
m \ddot{y}(t)=F(y(t))-2 \dot{y}(t)+2 \dot{w}(t), \quad t \in \mathbb{R},
$$

где функция $w(t)$ является суммой в точке $x=0$ “приходящих" волн из (1.1.8):

$$
\begin{gathered}
w(t) \equiv g_{+}(t)+f_{-}(-t), \quad t \in \mathbb{R}, \\
\dot{w}(t) \in L^{2}(0, \infty) .
\end{gathered}
$$

ДокАЗАТЕЛЬСтво. Формулы (1.3.4), (1.3.5) получаются из второго уравнения системы (1.1.1) при подстановке выражений для $u(x, t)$ через функцию $y(t) \equiv u(0, t)$ и приходящие волны:

$$
u(x, t) \equiv \begin{cases}y(t-x)+g_{+}(x+t)-g_{+}(t-x) & \text { при } x>0, \\ y(t+x)+f_{-}(x-t)-f_{-}(-x-t) & \text { при } x<0 .\end{cases}
$$

В свою очередь эти формулы вытекают из выражений для уходяших волн $f_{+}(x-t)$, $g_{-}(x+t)$ через приходяшие волны $g_{+}(x+t), f_{-}(x-t)$ :

$$
y(t) \equiv f_{+}(-t)+g_{+}(t) \equiv f_{-}(-t)+g_{-}(t), \quad t \in \mathbb{R} .
$$

Наконец, (1.3.6) следует из (1.3.5) и (1.3.2).

ЗАмЕчАния. і) Уравнение (1.3.4) содержит диссипацию энергии, вызванную взаимодействием струны и осциллятора. Описание обратимой системы (1.1.1) с помошњю “необратимого” уравнения (1.3.4) представляется парадоксальным. Объяснение парадокса следующее: наряду с (1.3.4) справедливо аналогичное уравнение с "отрицательным" трением, но с “уходяшими" волнами $w(t)$ вместо “приходящих" волн в правой части (1.3.4). Следовательно, эти уравнения меняются ролями, т.е. переходят друг в друга при обрашении времени. Таким образом, уравнение (1.3.4) связано с обратимой системой (1.1.1) “ковариантно” относительно инверсии времени.

ii) Для получения асимптотики решений системы (1.1.1) при $t \rightarrow+\infty$ необходимо использовать уравнение (1.3.4) с положительным трением и с приходящими волнами. Дело в том, что приходящие волны определяются непосредственно по начальным данным, в противоположность уходяшим волнам. 
iii) Связь меж ду уравнением (1.3.4) и системой (1.1.1) с несколько другой точки зрения изучалась в [24]. Для случая линейного осциллятора, когда $F(y) \equiv-k y, k>0$, система (1.1.1) и уравнение (1.3.4) впервые рассматривались Г. Лэмбом [41].

Согласно (1.3.2) входяшие в (1.3.4) функции, известные из (1.3.1), удовлетворяют условиям $f_{-}^{\prime}(-t), g_{+}^{\prime}(t) \in L^{2}\left(\mathbb{R}_{+}, \mathbb{R}^{d}\right)$. С другой стороны, из предположения о сушествовании решения вытекает согласно (1.1.11), что $\ddot{y}(t) \in L_{\mathrm{loc}}^{2}\left(\overline{\mathbb{R}}_{+}, \mathbb{R}^{d}\right)$. По определению 1.1.2 производная $\ddot{y}$ в (1.3.4) понимается в смысле обобщенных функций. Следовательно, $y \in C^{1}\left(\overline{\mathbb{R}}_{+}, \mathbb{R}^{d}\right)$ и по теореме Лебега уравнение (1.3.4) эквивалентно тому же тождеству при почти всех $t>0$. Поэтому при любых фиксированных начальных данных $y(0+)$ и $\dot{y}(0+)$ решение $y(t)$ уравнения $(1.3 .4)$ сушествует и единственно на некотором интервале $t \in[0, \varepsilon)$, где $\varepsilon>0$, так как $F(\cdot) \in C^{1}\left(\mathbb{R}^{d}, \mathbb{R}^{d}\right)$. Это легко доказать методом сжимаюших отображений, записывая (1.3.4) в эквивалентной интегральной форме

$$
m y(t)=\int_{0}^{t}\left(\int_{0}^{s} F(y(\tau)) d \tau\right) d s+2 \int_{0}^{t}[w(s)-y(s)] d s+C_{0}+C_{1} t, \quad t>0
$$

где

$$
C_{0}=m y(0+) \text { и } C_{1}=-2[w(0+)-y(0+)]+m \dot{y}(0+) .
$$

Итак, $y(t)$ единственным образом определяется на $[0, \varepsilon)$ при некотором $\varepsilon>0$. Поскольку $y \in C^{1}\left(\overline{\mathbb{R}}_{+}, \mathbb{R}^{d}\right)$, то $y(t)$ единственным образом определяется также и при всех $t>0$ для любых фиксированных $y(0+)$ и $\dot{y}(0+)$.

Остается уточнить выбор $y(0+)$ и $\dot{y}(0+)$. Во-первых, из (1.1.2) вытекает, что

$$
\dot{y}(0+)=p^{0} .
$$

Далее, из непрерьвности $u(x, t)$ при $|x|=t$ и из (1.3.2) вытекает непрерьвность $f_{+}(x-t)$ при $x=t$ и непрерьвность $g_{-}(x+t)$ при $x=-t$. Следовательно, ввиду (1.3.1) имеем

$$
\left\{\begin{array}{l}
f_{+}(0-)=f_{+}(0+) \equiv \frac{u^{0}(0)}{2}, \\
g_{-}(0+)=g_{-}(0-) \equiv \frac{u^{0}(0)}{2} .
\end{array}\right.
$$

Оба эти условия эквивалентны тому, что

$$
y(0+)=f_{ \pm}(0-)+g_{ \pm}(0+)=u^{0}(0) .
$$

Итак, $y(0+)$ и $\dot{y}(0+)$ определяются единственным образом, а с ними и $y(t)$ при $t \geqslant 0$. Затем $f_{+}$и $g_{-}$находятся однозначно из (1.3.8). Таким образом, единственность решения $u(x, t)$ при $t \geqslant 0$ доказана. Аналогично доказывается единственность и при $t \leqslant 0$.

Существование решений. Докажем существование решения $Y(t) \in C(\mathbb{R}, \mathscr{E})$ задачи (1.1.7). Прежде всего определим $u(x, t)$ в области $|x| \geqslant|t|$ формулой Даламбера (1.3.3). Тогда $u(x, t)$ ввиду (1.3.2) соответствует определению класса решений $C(\mathbb{R}, \mathscr{E})$ в области $|x|>|t|$.

Теперь построим решение в области $t>0,|x|<t$ : определим $u(x, t)$ формулой (1.1.8), в которой $f_{+}$и $g_{-}$находятся из (1.3.8) по функции $y(t)$. Здесь $y(t)-$ решение уравнения (1.3.4) с начальными условиями $(1.3 .10),(1.3 .12)$. 
ЛЕмма 1.3.2. Для любъх $у(0+)$ и $\dot{y}(0+)$ уравнение (1.3.4) имеет единственное решение при всех $t>0, u$

$$
\sup _{t>0}\left|y^{(k)}(t)\right| \leqslant y^{k}, \quad k=0,1
$$

где $y^{k}$ ограничены при ограниченных $\left\|\left(u^{0}, v^{0}, p^{0}\right)\right\|_{\mathscr{E}}$.

ДокАЗАТЕЛЬСтво. Выведем априорную оценку для $y(t)$. Для этого умножим (1.3.4) на $\dot{y}(t)$ для почти всех $t>0$, и, учитывая $F(y)=-\nabla V(y)$, получим

$$
m \frac{d}{d t} \frac{\dot{y}^{2}(t)}{2}=-\frac{d}{d t} V(y(t))+2[\dot{w}(t)-\dot{y}(t)] \dot{y}(t)
$$

для почти всех $t>0$. Интегрируя это равенство, из (1.3.6) и неравенства КошиШварца получим

$$
m \frac{\dot{y}^{2}(t)}{2}+V(y(t)) \leqslant B, \quad t>0
$$

где $B$ ограничено при ограниченных $\left\|\left(u^{0}, v^{0}, p^{0}\right)\right\|_{\mathscr{E}}$ в силу (1.3.2). Следовательно, из (0.1.15) следует (1.3.13) с $k=1$.

Из этой оценки вытекает существование и единственность глобального решения (1.3.4) при любых $y(0+)$ и $\dot{y}(0+)$, оценка (1.1.13), а также включение

$$
\dot{y}(t) \in C\left(\overline{\mathbb{R}}_{+}, \mathbb{R}^{d}\right) .
$$

Итак, определим $f_{+}$и $g_{-}$из (1.3.8) по построенной функции $y(t)$. Тогда из (1.3.16), (1.3.2) и (1.3.8) вытекает, что

$$
f_{+}^{\prime}(z) \in L_{\mathrm{loc}}^{2}\left(\overline{\mathbb{R}}_{-}, \mathbb{R}^{d}\right), \quad g_{-}^{\prime}(z) \in L_{\mathrm{loc}}^{2}\left(\overline{\mathbb{R}}_{+}, \mathbb{R}^{d}\right)
$$

Поэтому функция $u(x, t)$, определенная при $t>0$ по формуле (1.1.8), соответствует определению класса решений $C(\mathbb{R}, \mathscr{E})$ в области $|x|<t$. Следовательно, из (1.3.12) и (1.3.11) вытекает, что при $t>0$ функция $Y(t)=(u(x, t), \dot{u}(x, t), \dot{y}(t))$ есть ограничение на область $t>0$ некоторой функции из $C(\mathbb{R}, \mathscr{E})$.

Аналогично строится решение $u(x, t)$ и в области $t<0$. Из (1.3.3) следует непрерьвность построенной функции $Y(t)$ при $t=0$; и, следовательно, $Y(t) \in C(\mathbb{R}, \mathscr{E})$.

Остается проверить, что построенная функция $u(x, t)$ является решением задачи (1.1.1), (1.1.2). Из (1.1.8) следует, что $u(x, t)$ удовлетворяет первому уравнению (1.1.1). Начальные условия (1.1.2) для $u$ вытекают из (1.3.3) и (1.3.10). Из построения $f_{+}$и $g_{-}$видно, что $u(x, t)$ удовлетворяет второму уравнению (1.1.1) для $t>0$ (и при $t<0$ аналогично). Второе уравнение в (1.1.1) следует из уравнения (1.3.4) при $t>0$ и аналогичного уравнения при $t<0$, а также из равенства $\dot{y}(0+)=p^{0}=\dot{y}(0-)$ и включения $\ddot{y}(t) \in L_{\mathrm{loc}}^{2}\left(\overline{\mathbb{R}}_{ \pm}, \mathbb{R}^{d}\right)$ (последнее следует из (1.3.4), (1.3.16), (1.3.2)). Таким образом, первый пункт предложения 1.2.1 доказан. 
1.3.2. Непрерывность динамики. Изприведенного построения вьводится также второе утверждение предложения 1.2.1. Для этого достаточно доказать следуюшую лемму о непрерывной зависимости решений уравнения (1.3.4) от начальных данных из (1.1.2).

Лемма 1.3.3. Пусть $y_{1}(t)$ и $y_{2}(t)$ - решения уравнения (1.3.4) с начальным.м данными $\left(u_{i}^{0}, v_{i}^{0}, p_{i}^{0}\right), i=1,2$, соответственно. Тогда для любого $T>0$ существует такая константа $C_{T}$, ограниченная при ограниченных $\left\|\left(u_{i}^{0}, v_{i}^{0}, p_{i}^{0}\right)\right\|_{\mathscr{E}}$, что

$$
\begin{aligned}
& \sup _{[0, T]}\left|y_{2}(t)-y_{1}(t)\right| \leqslant C_{T}\left\|\left(u_{2}^{0}-u_{1}^{0}, v_{2}^{0}-v_{1}^{0}, p_{2}^{0}-p_{1}^{0}\right)\right\|_{\mathscr{E}}, \\
& \sup _{[0, T]}\left|\dot{y}_{2}(t)-\dot{y}_{1}(t)\right| \leqslant C_{T}\left\|\left(u_{2}^{0}-u_{1}^{0}, v_{2}^{0}-v_{1}^{0}, p_{2}^{0}-p_{1}^{0}\right)\right\|_{\mathscr{E}} .
\end{aligned}
$$

ДокАЗАТЕЛЬСтво. Для доказательства оценки (1.3.18) достаточно вычесть уравнение (1.3.9) для $y_{1}$ из такого же уравнения для $y_{2}$ и применить неравенство Гронуолла. Мы также используем априорную оценку (1.3.13). Дифференцируя (1.3.9), получаем оценку (1.3.19).

Из этой леммы и представления (1.3.7) вытекает непрерывность оператора $W_{t}$ в $\mathscr{E}$. Поэтому $W_{t}$ непрерьвен также и в $\mathscr{E}_{F}$, так как $u(x, t)$ зависит только от начальных данных $\left(u^{0}(y), v^{0}(y), p^{0}\right)$ с $|y-x|<|t|$ в силу (1.3.7) и (1.3.9).

1.3.3. Сохранение энергии. Сначала докажем закон сохранения энергии (1.1.3) для достаточно гладких начальных данных, когда $u^{0}(x) \in C^{2}\left(\mathbb{R} \backslash\{0\}, \mathbb{R}^{d}\right)$ и $v^{0}(x) \in$ $C^{1}\left(\mathbb{R} \backslash\{0\}, \mathbb{R}^{d}\right)$, причем сушествуют пределы $u^{0}(0 \pm),\left(u^{0}\right)^{\prime}(0 \pm), v^{0}(0 \pm)$ и, кроме того, (1.2.3) вьполняется с некоторой константой $r^{0}$. Заметим, что такие достаточно гладкие начальные данные образуют всюду плотное множество в банаховом пространстве $\mathscr{E}$ с нормой (1.1.4).

Из приведенного вьшше построения решения $u(x, t)$ видно, что для таких начальных данных $u \in C\left(\mathbb{R}^{2}, \mathbb{R}^{d}\right)$ все первые и вторые частные производные от $u(x, t)$ существуют в обычном смысле и локально ограничены при $x \neq 0$ и $x \neq \pm t$. Кроме того, при этом для всех $t \in \mathbb{R}$ сушествуют односторонние пределы $\dot{u}(x, t)$ и $u^{\prime}(x, t)$ при $x \rightarrow 0 \pm$ и $x \rightarrow t \pm 0$, причем

$$
\left.\left(\dot{u}+u^{\prime}\right)\right|_{x=t-0}=\left.\left(\dot{u}+u^{\prime}\right)\right|_{x=t+0} \quad \forall t \neq 0 .
$$

Это вытекает из разложения Даламбера (1.1.8). Действительно, обе части (1.3.20) для функции $f_{ \pm}(x-t)$ равны нулю, а функции $g_{ \pm}(x+t)$ непрерьвно дифференцируемы при $x=t \neq 0$. Аналогично,

$$
\left.\left(\dot{u}-u^{\prime}\right)\right|_{x=-t-0}=\left.\left(\dot{u}-u^{\prime}\right)\right|_{x=-t+0} \quad \forall t \neq 0 .
$$

Рассмотрим "интеграл энергии"

$$
I(t) \equiv \int_{-\infty}^{+\infty}\left[\frac{|\dot{u}(x, t)|^{2}}{2}+\frac{\left|u^{\prime}(x, t)\right|^{2}}{2}\right] d x
$$


при $t>0$. Разобъем его на сумму интегралов по интервалам $(-\infty,-t),(-t, 0),(0, t)$ и $(t, \infty)$. Тогда, дифференцируя по $t$ каждьй из этих интегралов, получим

$$
I^{\prime}(t)=\left.\Gamma_{-}(x, t)\right|_{x=-t+0} ^{x=-t-0}+\left.u^{\prime} \dot{u}\right|_{x=0+} ^{x=0-}+\left.\Gamma_{+}(x, t)\right|_{x=t+0} ^{x=t-0}
$$

где

$$
\Gamma_{ \pm}(x, t)= \pm\left[\frac{|\dot{u}(x, t)|^{2}}{2}+\frac{\left|u^{\prime}(x, t)\right|^{2}}{2}\right]+u^{\prime}(x, t) \dot{u}(x, t)= \pm \frac{1}{2}\left|\dot{u}(x, t) \pm u^{\prime}(x, t)\right|^{2} .
$$

Здесь использовано, что уравнение (1.1.1) вьполняется для $u(x, t)$ при $x \neq 0$ и $x \neq \pm t$ в обычном смысле. Из (1.3.20) и (1.3.21) вытекает, что

$$
\left.\Gamma_{ \pm}\right|_{x= \pm t+0} ^{x= \pm t-0}=0
$$

Наконец, из уравнения (1.1.1) получаем, что

$$
\left.u^{\prime} \dot{u}\right|_{x=0+} ^{x=0-}=-\dot{y}\left(m \ddot{y}+V^{\prime}(y)\right)=-\frac{d}{d t}\left[m \frac{\dot{y}^{2}}{2}+V(y(t))\right], \quad t>0 .
$$

Поэтому из (1.3.23) вытекает, что

$$
I^{\prime}(t)+\frac{d}{d t}\left[m \frac{\dot{y}^{2}}{2}+V(y(t))\right]=0, \quad t>0 .
$$

Отсюда следует $(E)$ для $t>0$. Тогда предельным переходом $t \rightarrow 0+$ получаем $(E)$ и при $t \geqslant 0$. При $t \leqslant 0$ тождество $(E)$ доказьвается так же. Итак, тождество $(E)$ доказано для всюду плотного множества начальных данных из $\mathscr{E}$. Остается сослаться на предложение 1.2.1 ii).

\section{§ 1.4. Релаксация для приведенного уравнения}

Мы выведем теорему 1.2.2 в следуюшем параграфе из представления (1.3.7) и из следуюшей леммы о релаксации для приведенного уравнения. Обозначим $\mathscr{Z}=\left\{(z, 0) \in \mathbb{R}^{2 d}: z \in Z\right\}$.

ЛЕмма 1.4.1. Пусть выполнены условия теоремы 1.2.2. Тогда справедливы следующие утверәсдения.

i) Конечен интеграл рассеяния

$$
\int_{0}^{\infty}|\dot{y}(t)|^{2} d t<\infty
$$

ii) Для любого решения $y(t)$ уравнения (1.3.4)

$$
(y(t), \dot{y}(t)) \rightarrow \mathscr{Z} \quad \text { npu } \quad t \rightarrow \infty .
$$

iii) Пусть, дополнительно, $Z$ - захватьввающее подмножество в $\mathbb{R}^{d}$. Тогда существует такая точка $(z, 0) \in \mathscr{Z}$, что

$$
(y(t), \dot{y}(t)) \rightarrow(z, 0) \quad \text { npu } \quad t \rightarrow \infty .
$$

iv) Ecли, кроме того, $d=1 u \dot{w}(t) \equiv 0$ при $t>t^{0}$, то (1.4.3) справедливо при любом множестве $Z$.

Прежде чем доказывать эту лемму, приведем типичные примеры для $d=1$. 
1.4.1. Примеры. Для простоты допустим, что начальные данные удовлетворяют условию (1.2.3). Тогда из (1.3.5) следует $\dot{w}(t) \equiv 0$ для $t>r^{0}$, и уравнение (1.3.4) является автономным для больших $t$.

Невырожденные потенциалы. Сначала рассмотрим случай $m>0$. Тогда на фазовой плоскости $(y, \dot{y})$ фазовые кривые приведенного уравнения (1.3.4) описываются следующей системой:

$$
\left\{\begin{array}{rl}
\dot{y}(t) & =v(t), \\
m \dot{v}(t) & =F(y(t))-2 \nu v(t),
\end{array} \quad t>r^{0} .\right.
$$

Здесь $\nu=1$ для системы (1.1.1) и в более общем случае $\nu=\sqrt{\mu T}$, где $\mu$ - линейная плотность струны и $T$ - ее натяжение. Рассмотрим эту систему как возмушение системы с $\nu=0$, соответствуюшей свободному осциллятору, не связанному со струной,

$$
\left\{\begin{aligned}
\dot{y} & =v, \\
m \dot{v} & =F(y) .
\end{aligned}\right.
$$

Установим некоторые простые связи между фазовыми портретами этих двух систем.

А. Стационарные точки этих систем совпадают.

В. Вертикальная компонента $\dot{v}$ вектора фазовой скорости в системе (1.4.4) меньше, чем в (1.4.5) в верхней полуплоскости $v>0$, и больше в нижней полуплоскости $v<0$. В то же время горизонтальные компоненты этих векторов равны (рис. 2).

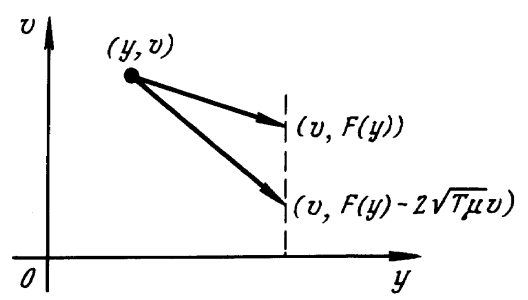

Рис. 2

C. Поэтому фазовые кривые системы (1.4.4) пересекают фазовые кривые системы (1.4.5) сверху вниз в полуплоскости $v>0$ и снизу вверх в полуплоскости $v<0$.

Рассмотрим типичный пример невырожденного потенциала

$$
V(y)=\frac{y^{4}}{4}-\frac{y^{2}}{2}, \quad y \in \mathbb{R} .
$$

Этот потенциал удовлетворяет условиям (0.1.15). При этом система (1.4.5) имеет следуюшие фазовые кривые:

- замкнутые кривые, соответствуюшие периодическим решениям,

- две сепаратрисы, входящие в точку $(0,0)$ и выходящие из нее же,

- три стационарные точки: седло в точке $(0,0)$ и два центра в точках $( \pm 1,0)$ (рис. 3). 


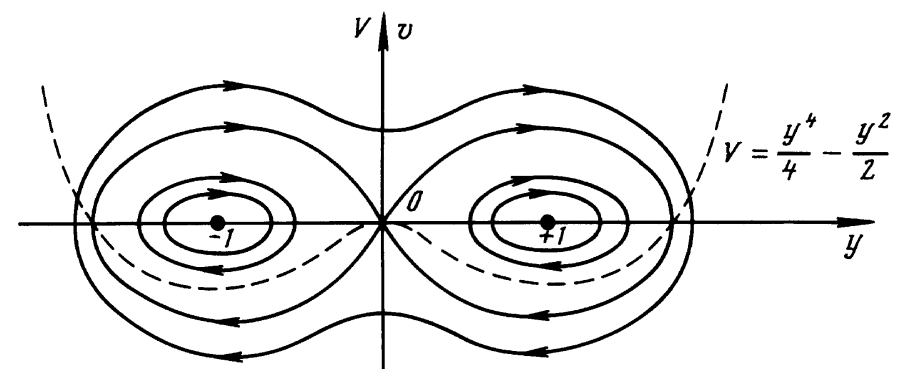

Рис. 3

Учитьвая свойство С, получаем, что система (1.4.4) с потенциалом (1.4.6) имеет

- устойчивые фокусы в точках $( \pm 1,0)$ при малых $\nu>0$ (устойчивые узлы при больших $\nu>0)$,

- седло в точке $(0,0)$ (рис. 4$)$.

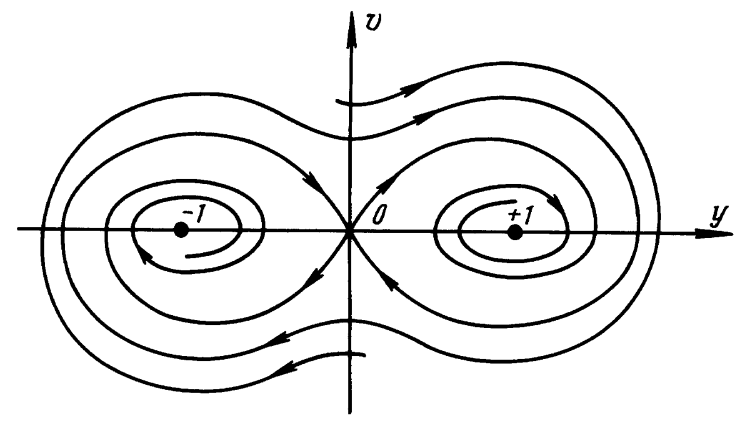

Рис. 4

Теперь рассмотрим случай $m=0$. Тогда для потенциала (1.4.6) фазовые кривые системы (1.4.4) - это лучи $y<-1, y>1$, интервалы $(-1,0),(0,+1)$ и три стационарные точки (устойчивые точки \pm 1 и неустойчивая точка 0 ) (рис. 5 ).

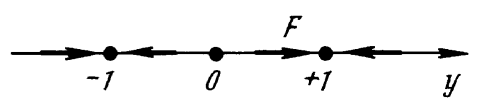

Рис. 5

ЗАмЕчАниЕ. В рассмотренном примере (потенциал (1.4.6) для $m>0$ ) условия леммы 1.4.1 выполнены и ее утверждения справедливы. В частности, все фазовые кривые при $t \rightarrow+\infty$ входят в какую-нибудь стационарную точку. Отметим, что эта предельная точка может быть и неустойчивой, как, например, седловая точка $(0,0)$ на рис. 4, в которую входят две сепаратрисы. 
Вырожденные потенциалы. Пример вырожденного потеншиала дает функция

$$
V(y) \equiv \begin{cases}k \frac{\left(y-b_{1}\right)^{2}}{2}, & y \leqslant b_{1}, \\ 0, & b_{1} \leqslant y \leqslant b_{2}, \\ k \frac{\left(y-b_{2}\right)^{2}}{2}, & y \geqslant b_{2},\end{cases}
$$

где $k>0$ и $b_{1}<b_{2}$. В полосе $b_{1} \leqslant y \leqslant b_{2}$ фазовые кривые описьваются системой (1.4.4) с $F \equiv 0$, т.е.

$$
\left\{\begin{array}{rl}
\dot{y} & =v \\
m \dot{v} & =-2 \nu v
\end{array} \Rightarrow \frac{d v}{d y}=-\alpha \equiv-\frac{2 \nu}{m} .\right.
$$

Поэтому уравнения фазовых кривых в полосе $b_{1} \leqslant y \leqslant b_{2}$ имеют вид

$$
v=-\alpha y+\text { const } .
$$

В частности, при $\nu=0$ фазовые кривые - это отрезки прямых, параллельные оси $y$.

Учитьвая вышесказанное, получаем фазовые портреты системы (1.4.4) с потенциалом (1.4.7).

1) При $\nu=0$ (см. рис. 6).

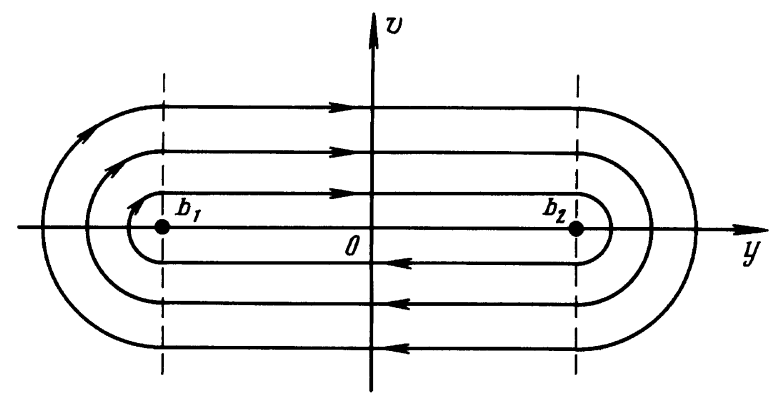

Рис. 6

Из рисунка видно, что в случае 1) утверждения ii) и іii) леммы 1.4.1 не выполняются. Осциллятор при $t \rightarrow+\infty$ совершает периодические колебания.

2) Для малых $\nu>0$ (см. рис. 7).

Из рисунка видно, что в случае 2) (и для больших $\nu>0$ также) все решения имеют пределы при $t \rightarrow+\infty$, что соответствует лемме 1.4.1 iv). Отметим, что потенциал (1.4.7) не удовлетворяет условию $(N D)$.

1.4.2. Доказательство релаксации для приведенного уравнения. Докажем лемму 1.4.1. 


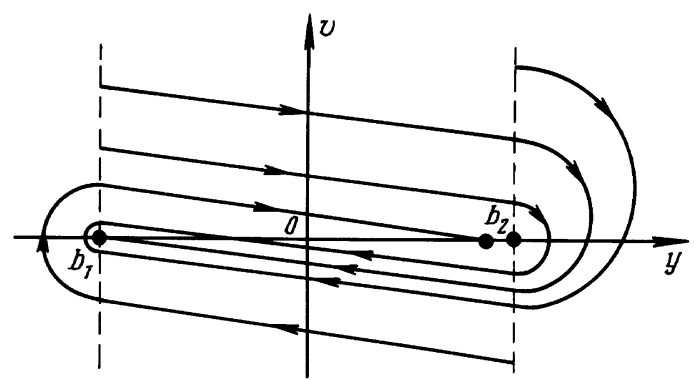

Рис. 7

i) Из (1.3.4) вытекает энергетическое тождество

$$
\frac{d}{d t}\left[m \frac{|\dot{y}(t)|^{2}}{2}+V(y(t))\right]=-2|\dot{y}(t)|^{2}+2 \dot{w}(t) \dot{y}(t), \quad t \in \mathbb{R} .
$$

Мажорируя правую часть функцией $-\varepsilon \dot{y}^{2}(t)+C_{\varepsilon} \dot{w}^{2}(t)$ с некоторыми $\varepsilon, C_{\varepsilon}>0$ и интегрируя, получаем

$$
\frac{\dot{y}^{2}(t)}{2}+V(y(t))+\varepsilon \int_{0}^{t} \dot{y}^{2}(\tau) d \tau \leqslant C_{0}+C_{\varepsilon} \int_{0}^{t} \dot{w}^{2}(\tau) d \tau \text { при } t>0 .
$$

Поэтому из (0.1.15) и (1.3.6) следует (1.4.1).

ii) Из (1.3.13) следует, что траектория $(y(t), \dot{y}(t))$ ограничена. Следовательно, остается доказать, что $(\bar{y}, \bar{p}) \in \mathscr{Z}$, если $\left(y\left(t_{k}\right), \dot{y}\left(t_{k}\right)\right) \rightarrow(\bar{y}, \bar{p})$ для некоторой последовательности $t_{k} \rightarrow \infty$. Докажем это от противного: предположим, что $(\bar{y}, \bar{p}) \notin \mathscr{Z}$. Сначала разберем случай $\bar{p} \neq 0$. Из (1.4.11) вытекает, что правая часть (1.4.10) суммируема на интервале $(0, \infty)$. Следовательно, сушествует предел

$$
E_{\infty} \equiv \lim _{t \rightarrow+\infty}\left[m \frac{|\dot{y}(t)|^{2}}{2}+V(y(t))\right]
$$

Тогда из $\bar{p} \neq 0$ вытекает $V(\bar{y})<E_{\infty}$. Следовательно, для некоторого $h>0$ мы имеем $V(y)<E_{\infty}-h$ в малой окрестности $U$ предельной точки $(\bar{y}, \bar{p})$. Поэтому из $(1.4 .12)$ вытекает, что для больших $t$

$$
m \frac{|\dot{y}(t)|^{2}}{2} \geqslant h>0 \quad \text { при } \quad(y(t), \dot{y}(t)) \in U
$$

Траектория $(y(t), \dot{y}(t))$ посещает $U$ бесконечно много раз. Введем моменты входа и выхода

$$
\tau_{k}^{-}=\inf \left\{t \in\left[0, t_{k}\right]:(y(s), \dot{y}(s)) \in U \text { при всех } s \in\left(t, t_{k}\right]\right\}
$$

$$
\tau_{k}^{+}=\sup \left\{t \in\left[t_{k}, \infty\right):(y(s), \dot{y}(s)) \in U \text { при всех } s \in\left(t_{k}, t\right]\right\} .
$$


Беря подпоследовательность, мы можем считать, что $t_{k+1}-t_{k}>2$ для каждого $k=1,2, \ldots$, и определим $t_{k}^{-}=\max \left(\tau_{k}^{-}, t_{k}-1\right), t_{k}^{+}=\min \left(\tau_{k}^{+}, t_{k}+1\right)$. Тогда отрезки $\left[t_{k}^{-}, t_{k}^{+}\right]$попарно не пересекаются и $(y(t), \dot{y}(t)) \in U$ при $t \in\left[t_{k}^{-}, t_{k}^{+}\right]$. Назовем промежуток $\left[t_{k}^{-}, t_{k}^{+}\right]$интервалом $k$-го посешения. При больших $k$ точка $\left(y\left(t_{k}\right), \dot{y}\left(t_{k}\right)\right)$ очень близка к $(\bar{y}, \bar{p})$ и тогда время пребьвания $t_{k}^{+}-t_{k}^{-} \geqslant T=\min \left(1, C / y^{1}\right)>0$ в силу (1.3.13) с $k=1$. Поэтому из (1.4.13) следует, что вклад каждого посешения в интеграл рассеяния (1.4.1) допускает оценку снизу,

$$
\frac{m}{2} \int_{t_{k}^{-}}^{t_{k}^{+}}|\dot{y}(t)|^{2} d t \geqslant h T>0 .
$$

Однако число посешений бесконечно, следовательно, (1.4.1) не может вьполняться, и это противоречие доказьвает, что $\bar{p}=0$.

Наконец, $\nabla V(\bar{y})=0$, что вытекает из аналогичного анализа посещений. Действительно, из $\nabla V(\bar{y}) \neq 0$ можно вывести оценку снизу типа (1.4.16) для вклада каждого посещения в интеграл рассеяния (1.4.1). Это следует из уравнения (1.3.4) ввиду (1.4.1).

iii) Из (1.4.2) вытекает (1.4.3), так как $Z$ - захватывающее подмножество в $\mathbb{R}$, и, следовательно, $\mathscr{Z}$ - захватывающее подмножество в $\mathbb{R}^{2 d}$.

iv) Если $\dot{w}(t) \equiv 0$ при $t>t^{0}$, то уравнение (1.3.4) принимает вид

$$
m \ddot{y}(t)=F(y(t))-2 \dot{y}(t), \quad t>t^{0} .
$$

Докажем (1.4.3) от противного. Если $y(t)$ не имеет предела, то

$$
y_{-}=\liminf _{t \rightarrow+\infty} y(t)<y_{+}=\limsup _{t \rightarrow+\infty} y(t) .
$$

Поэтому из (1.4.12) и $\dot{y}(t) \rightarrow 0$ вытекает $V(y)=E_{\infty}$ при $y_{-} \leqslant y \leqslant y_{+}$. Следовательно,

$$
m \ddot{y}(t)=-2 \dot{y}(t), \text { если } y_{-}<y(t)<y_{+},
$$

и фазовые кривые в полосе $y_{-}<y<y_{+}$плоскости $y, \dot{y}$ являются линиями

$$
\dot{y}=-\frac{2}{m} y+\text { const } .
$$

Поэтому из $\dot{y}(t) \rightarrow 0$ следует (1.4.3) (см. рис. 7$)$.

\section{$\S 1.5$. Долговременная асимптотика}

1.5.1. Переходы к стационарным состояниям. Мы доказываем здесь теоремy 1.2.2.

Компакное притягивающее множество. Сначала построим компактное притягиваюшее множество $\mathscr{A}$ для рассматриваемой траектории $Y(t)$. 
ОПРЕДЕЛЕНИЕ 1.5.1. $\mathscr{A}=\left\{S_{z}: z \in \mathbb{R}^{d},|z| \leqslant y^{0}\right\}$, где $S_{z}$ определены согласно (1.1.14), а $y^{0}$ - согласно (1.3.13).

Множество $\mathscr{A}$ компактно в $\mathscr{E}_{F}$, поскольку оно гомеоморфно компакту в $\mathbb{R}^{d}$.

Лемма 1.5.2. Пусть все условия теоремы 1.2 .2 выполнены. Тогда $Y(t) \stackrel{\mathscr{E}}{\longrightarrow} \mathscr{A}$ npu $t \rightarrow \pm \infty$.

ДокАЗАТЕЛЬСтво. Достаточно проверить, что для любого $R>0$ (cp. (0.1.14))

$$
\left.\left\|Y(t)-S_{y(t)}\right\|\right|_{R}=\|\left. u^{\prime}(\cdot, t)\right|_{R}+|\dot{u}(\cdot, t)|_{R}+|\dot{y}(t)| \rightarrow 0 \text { при } t \rightarrow \infty .
$$

Здесь полунормы $|\cdot|_{R}$ сходятся к нулю при $t \rightarrow \infty$ в силу (1.3.7), (1.3.2) и (1.4.1). Следовательно, (1.4.2) завершает доказательство.

ДоКАЗАТЕЛЬСТВо ТЕОРЕМЫ 1.2.2. і) Из леммы 1.5.2 вытекает, что орбита $O(Y)$ предкомпактна в $\mathscr{E}_{F}$. Поэтому из следующей леммы вытекает (1.2.1). Обозначим через $\Omega(Y)$ омега-предельное множество траектории $Y(t)$ в топологии $Ф$ реше пространства $\mathscr{E}_{F}: \bar{Y} \in \Omega(Y)$ тогда и только тогда, когда $Y\left(t_{k}\right) \stackrel{\mathscr{E}_{F}}{\longrightarrow} \bar{Y}$ для некоторой последовательности $t_{k} \rightarrow \pm \infty$.

ЛЕмма 1.5.3. $\Omega(Y)$ содержится в $\mathscr{S}$.

ДокАЗАТЕЛьСтво. $\Omega(Y) \subset \mathscr{A}$, поскольку $\mathscr{A}$ притягивающее множество. Кроме того, множество $\Omega(Y)$ инвариантно относительно $W_{t}, t \in \mathbb{R}$, в силу непрерьвности $W_{t}$ в $\mathscr{E}_{F}$. Поэтому для каждого $\bar{Y} \in \Omega(Y)$ сушествует $C^{2}$-кривая $t \mapsto z(t) \in \mathbb{R}^{d}$, для которой $W_{t} \bar{Y}=S_{z(t)}$. Это значит, что $S_{z(t)}$ - решение системы (1.1.7). Но тогда $\dot{z}(t)=0$, т.е. $z(t) \equiv z$ и $\bar{Y}=S_{z} \in S$.

ii) Из представления (1.3.7), (1.3.2) и леммы 1.4.1 i), iii) вытекает (1.2.2).

iii) Из (1.2.3) следует $\dot{w}(t) \equiv 0$ при $t>r^{0}$. Тогда в силу (1.2.3) и представления (1.3.7)

$$
u(x, t)=y(t-|x|) \text { при } t-|x|>r^{0} .
$$

Следовательно, из леммы 1.4.1 вытекает (1.2.2).

1.5.2. Затухающие блуждания. Выведем лемму 1.2.3 из формул (1.3.4)-(1.3.7). Допустим, что $(N D)$ не вьполняется. Пусть, без ограничения обшности,

$$
F(z) \equiv 0 \text { при }-1 \leqslant z \leqslant 1 .
$$

Рассмотрим случаи $m=0$ и $m \neq 0$ отдельно.

I. Если $m=0$, то в качестве $y(t)$ можно взять произвольную функцию со значениями в отрезке $[-1,1]$, для которой $\dot{y}(t) \in L^{2}(\mathbb{R})$, и положить $u(x, t)=y(t-x)$. Тогда $Y(t)=(u(\cdot, t), \dot{u}(\cdot, t)) \in C\left(\mathbb{R}, \mathscr{E}^{0}\right)$ - решение системы (1.1.1). Возьмем, например,

$$
y(t)=\sin \log \left(1+t^{2}\right) \text { при } t \in \mathbb{R} .
$$

Тогда (1.2.2) очевидно не выполняется.

II. Если $m \neq 0$, то возьмем $y(t)$ опять в виде (1.5.4), определим $\dot{w}(t)$ из (1.3.4) и положим $g_{+}(t) \equiv w(t), f_{-}(t) \equiv 0$. Тогда (1.3.4) выполняется, и $u(x, t)=y(t-|x|)$ согласно (1.3.7). Следовательно, $Y(t)=(u(\cdot, t), \dot{u}(\cdot, t), \dot{y}(t)) \in C(\mathbb{R}, \mathscr{E})$ - решение системы (1.1.1), и (1.2.2) очевидно не выполняется. 
1.5.3. Транзитивность. Докажем лемму 1.2.4. Пусть $S_{ \pm}=\left(s_{ \pm}(x), 0,0\right)$, где $s_{ \pm}(x) \equiv z_{ \pm} \in Z$. Переходы $S_{-} \rightarrow S_{+}$можно построить различными способами. Выберем наиболее простой. Построим решение $Y(t)=(u(\cdot, t), \dot{u}(\cdot, t), \dot{y}(t)) \in C(\mathbb{R}, \mathscr{E})$ системы (1.1.7), для которого

$$
y(t) \equiv u(0 \pm, t)=\left\{\begin{array}{l}
z_{-} \text {при } t \leqslant-1, \\
z_{+} \text {при } t \geqslant 1 .
\end{array}\right.
$$

Продолжим произвольно $y(t)$ на $t \in(-1,1)$ так, чтобы $y(t) \in C^{2}\left(\mathbb{R}, \mathbb{R}^{d}\right)$. Затем положим $g_{+} \equiv z_{-}$и определим $f_{-}$из (1.3.4):

$$
m \ddot{y}(t)=F(y(t))+2\left(f_{-}^{\prime}(-t)-\dot{y}(t)\right), \quad t \in \mathbb{R} .
$$

Тогда $f_{-}^{\prime}(z) \in C\left(\mathbb{R}, \mathbb{R}^{d}\right)$. Поскольку $F\left(z_{ \pm}\right)=0$, то

$$
f_{-}^{\prime}(-t) \equiv 0 \text { при } t \leqslant-1 \text { и при } t \geqslant 1 .
$$

Можно определить $f_{-}$однозначно, потребовав, например, чтобы

$$
f_{-}(-t) \equiv z_{-} \text {при } t \leqslant-1 .
$$

Тогда уходяшие волны $g_{-}$и $f_{+}$определяются из (1.3.8). Так как $y(t), f_{-}(-t)$ и $g_{+}(t)$ постоянны для больших $|t|$, то $f_{+}(-t), g_{-}(t)$ также постоянны для больших значений $|t|$. Поэтому для функции $u(x, t)$, определенной согласно (1.3.7), траектория $Y(t)=(u(\cdot, t), \dot{u}(\cdot, t), \dot{y}(t)) \in C(\mathbb{R}, \mathscr{E})$ является решением (1.1.7), и $(1.2 .2)$ вьполняется.

ЗАМЕчАнИя. і) Построенное решение означает, что осциллятор находится в стационарной точке $z_{-}$при $t \leqslant-1$; затем волна $f_{-}(x-t)$ падает на осциллятор и переводит его в состояние $z_{+}$к моменту $t=1$; кроме того, она при $t>-1$ порождает пару уходяших волн: $g_{-}(x+t)$ при $x<0$ и $f_{+}(x-t)$ при $x>0$. Эти волны бегут вдоль полосы $-1<t-|x|<1$.

ii) Физически, неравенство $z_{+} \neq z_{-}$означает захват излучения осциллятором, если $V\left(z_{+}\right)>V\left(z_{-}\right)$, или испускание излучения, если $V\left(z_{+}\right)<V\left(z_{-}\right)$.

\section{Глава II. Струна с конечньм числом нелинейных осцилляторов}

Рассматривается система уравнений, описьваюшая колебания бесконечной струны, связанной с конечным числом нелинейных осцилляторов. Доказывается сходимость к стационарным состояниям при $t \rightarrow \pm \infty$. В [31] и [32] этот результат получен при некоторых дополнительных ограничениях на начальные данные.

\section{§.1. Введение}

Мы устанавливаем сходимость типа $(0.1 .11),(0.1 .12)$ к стационарным состояниям для решений системы

$$
\left\{\begin{array}{l}
\ddot{u}(x, t)=u^{\prime \prime}(x, t), \quad x \in \mathbb{R} \backslash Q, \quad t \in \mathbb{R}, \\
u\left(x_{k}+0, t\right)=u\left(x_{k}-0, t\right), \quad t \in \mathbb{R}, \quad k=1, \ldots, N, \\
0=F_{k}\left(u\left(x_{k}, t\right)\right)+u^{\prime}\left(x_{k}+0, t\right)-u^{\prime}\left(x_{k}-0, t\right) .
\end{array}\right.
$$


Здесь $Q=\left\{x_{1}, \ldots, x_{N}\right\}-$ конечное множество из $N$ точек $x_{i} \in \mathbb{R}$. В случае $N=1$ система совпадает с системой Лэмба (1.1.1) при $m=0$. Решения $u(x, t)$ принимают значения в $\mathbb{R}^{d} c d \geqslant 1$. Мы рассматриваем задачу Коши для системы (2.1.1) с начальными условиями

$$
\left.u\right|_{t=0}=u^{0}(x),\left.\quad \dot{u}\right|_{t=0}=v^{0}(x), \quad x \in \mathbb{R}
$$

Система (2.1.1) формально эквивалентна одномерному нелинейному волновому уравнению с нелинейным членом $\sum_{k=1}^{N} \delta\left(x-x_{k}\right) F_{k}(u)$, сосредоточенным на множестве $Q$ (cp. (1.1.3)),

$$
\ddot{u}(x, t)=u^{\prime \prime}(x, t)+\sum_{k=1}^{N} \delta\left(x-x_{k}\right) F_{k}(u(x, t)), \quad(x, t) \in \mathbb{R}^{2} .
$$

Физически, система (2.1.1) описьвает малые поперечные колебания струны. На струну в точках $x_{k}$ действуют перпендикулярные к ней силы $F_{k}$. Например, $F_{k}=-R y_{k}$, если в точке $x_{k}$ к струне присоединена линейная пружина жесткости $R$ (рис. 8). Но, вообще говоря, функции $F_{k}\left(y_{k}\right)$ нелинейны.

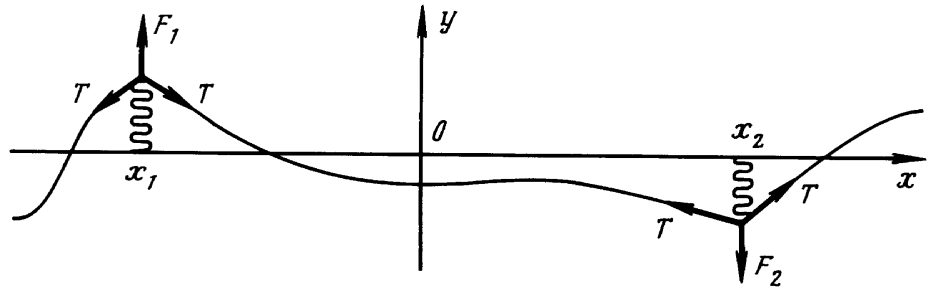

Рис. 8

Введем “конфигурационное пространство” $\mathscr{Q}$ и фазовое пространство $\mathscr{E}$ состояний конечной энергии для системы (2.1.1), совпадающее с пространством $\mathscr{E} 0$ из главы I. Обозначим через $L^{2}$ гильбертово пространство $L^{2}\left(\mathbb{R}, \mathbb{R}^{d}\right)$ с нормой $|\cdot|$ и и через $|\cdot|_{R}-$ норму в $L^{2}\left(-R, R ; \mathbb{R}^{d}\right)$ для $R>0$.

ОПРЕДЕЛЕНИЕ 2.1.1. і) $\mathscr{Q}$ - гильбертово пространство $\left\{u(x) \in C\left(\mathbb{R}, \mathbb{R}^{d}\right): u^{\prime}(x) \in\right.$ $\left.L^{2}\right\}$ с нормой

$$
\|u\|_{\mathscr{Q}}=\left|u^{\prime}\right|+|u(0)| .
$$

ii) $\mathscr{E}=\mathscr{Q} \oplus L^{2}$ - гильбертово пространство пар $(u(x), v(x))$ с нормой

$$
\|(u, v)\|_{\mathscr{E}}=\|u\|_{\mathscr{Q}}+|v| .
$$

iii) $\mathscr{E}_{F}-$ пространство $\mathscr{E}$, снабженное топологией Фреше, определяемой полунормаМИ

$$
\|(u, v)\|_{R} \equiv\left\|\left.u^{\prime}\right|_{R}+|u(0)|+\right\| v \|_{R}, \quad R>0 .
$$


Мы предполагаем вьполненными условия (0.1.16)-(0.1.17). При этом система (2.1.1) формально является гамильтоновой с фазовым пространством $\mathscr{E}$ и гамильтонианом

$$
\mathscr{H}(u, v)=\frac{1}{2} \int_{\mathbb{R}}\left[|v(x)|^{2}+\left|u^{\prime}(x)\right|^{2}\right] d x+\sum_{k=1}^{N} V_{k}\left(u\left(x_{k}\right)\right)
$$

для $(u, v) \in \mathscr{E}$. Мы рассматриваем решения $u(x, t)$, для которых $Y(t)=(u(\cdot, t), \dot{u}(\cdot, t))$ $\in C(\mathbb{R}, \mathscr{E})$, и записьваем задачу Коши (2.1.1)-(2.1.2) в виде

$$
\dot{Y}(t)=\mathscr{V}(Y(t)) \text { при } t \in \mathbb{R}, \quad Y(0)=Y^{0},
$$

где $Y^{0}=\left(u^{0}, v^{0}\right)$. Обсудим постановку этой задачи Коши для функций $Y(t) \in C(\mathbb{R}, \mathscr{E})$. Второе уравнение (2.1.1) имеет смысл и выполняется автоматически, так как $u \in$ $C\left(\mathbb{R}^{2}, \mathbb{R}^{d}\right)$, поскольку $Y(t) \in C(\mathbb{R}, \mathscr{E})$. Смысл третьего уравнения (2.1.1) в случае $N=1$ объяснен в главе І. Применим эти построения к системе (2.1.1). Уравнение (2.1.1) понимается в смысле распределений в области $x \in \mathbb{R} \backslash Q, t \in \mathbb{R}$. Поэтому оно эквивалентно разложению Даламбера для всех $k=1, \ldots, N+1$,

$$
u(x, t)=f_{k}(t-x)+g_{k}(t+x), \quad x \in \Delta_{k}, \quad t \in \mathbb{R},
$$

где $f_{k}, g_{k} \in C\left(\mathbb{R}, \mathbb{R}^{d}\right)$, так как $u \in C\left(\mathbb{R}^{2}, \mathbb{R}^{d}\right)$. Поэтому для всех $k=1, \ldots, N$ и почти всех $(x, t) \in \Delta_{k} \times \mathbb{R}$

$$
\dot{u}(x, t)=f_{k}^{\prime}(t-x)+g_{k}^{\prime}(t+x), \quad u^{\prime}(x, t)=-f_{k}^{\prime}(t-x)+g_{k}^{\prime}(t+x),
$$

где все производные понимаются в смысле распределений. Из условия $Y(t) \in C(\mathbb{R}, \mathscr{E})$ следует

$$
f_{k}^{\prime}(\cdot), g_{k}^{\prime}(\cdot) \in L_{\mathrm{loc}}^{2}\left(\mathbb{R}, \mathbb{R}^{d}\right) \quad \forall k=1, \ldots, N+1
$$

Теперь обсудим третье уравнение системы (2.1.1).

ОПРЕДЕЛЕНИЕ 2.1.2. В третьем уравнении системы (2.1.1) для всех $k=1, \ldots, N$

$$
\begin{aligned}
& u^{\prime}\left(x_{k}-0, t\right) \equiv-f_{k}^{\prime}\left(t-x_{k}\right)+g_{k}^{\prime}\left(t+x_{k}\right) \in L_{\mathrm{loc}}^{2}\left(\mathbb{R}, \mathbb{R}^{d}\right), \\
& u^{\prime}\left(x_{k}+0, t\right) \equiv-f_{k+1}^{\prime}\left(t-x_{k}\right)+g_{k+1}^{\prime}\left(t+x_{k}\right) \in L_{\mathrm{loc}}^{2}\left(\mathbb{R}, \mathbb{R}^{d}\right) .
\end{aligned}
$$

Заметим, что функции $f_{k}$ и $g_{k}$ в (2.1.9) определены с точностью до константы. Поэтому определение 2.1.12 однозначно. 


\section{§ 2.2. Основные результаты}

Начнем с построения динамики.

ПРЕДЛОЖЕНИЕ 2.2.1. Пусть $d \geqslant 1$ и выполнены условия (0.1.16)-(0.1.17). Тог$\partial a$

i) для любого $Y^{0} \in \mathscr{E}$ задача Коши (2.1.8) имеет единственное решение $Y(t) \in C(\mathbb{R}, \mathscr{E})$

ii) отобрахсение $W_{t}: Y^{0} \mapsto Y(t)$ непрерывно в $\mathscr{E}$ и $\mathscr{E}_{F}$ при любом $t \in \mathbb{R}$;

iii) выполняется закон сохранения энергии $(E)$.

Обозначим через $\mathscr{S}$ множество стационарных состояний $S=(s(x), 0) \in \mathscr{E}$ системы (2.1.8). Функции $s(x)$ линейны по $x$ на каждом интервале $\Delta_{k} \equiv\left(x_{k-1}, x_{k}\right)$, $k=2, \ldots, N$; они постоянны в $\Delta_{1} \equiv\left(-\infty, x_{1}\right)$ и в $\Delta_{N+1} \equiv\left(x_{N},+\infty\right)$. Следуюшее предложение дает критерий, когда множество $\mathscr{S}$ является непустым дискретным захватывающим подмножеством в $\mathscr{E}_{F}$.

ПРЕДЛОЖЕНИЕ 2.2.2. Пусть условия (0.1.16)-(0.1.17) выполнены и, кроме того, $d=1$ и все функиии $F_{k}(y), k=1, \ldots, N$, вещественно-аналитические на $\mathbb{R}$. Тогда $\mathscr{S}$ - дискретное захватывающее подмнохество в $\mathscr{E}_{F}$, и мнохество $\{S \in \mathscr{S}: \mathscr{H}(S) \leqslant h\}$ конечно для каждого $h \in \mathbb{R}$.

Главный результат этой главы означает, что множество $\mathscr{S}$ является точечным аттрактором системы $(2.1 .8)$ в топологии Фреше пространства $\mathscr{E}_{F}$.

ТЕОРема 2.2.3. Пусть выполнены все условия предложения 2.2 .2 и начальное состояние $Y^{0} \in \mathscr{E}$. Тогда

i) для решения $Y(t) \in C(\mathbb{R}, \mathscr{E})$ задачи Коши (2.1.8) орбита $O(Y)$ предкомпактна в $\mathscr{E}_{F}$ u

$$
Y(t) \stackrel{\mathscr{E}_{F}}{\longrightarrow} \mathscr{S} \quad \text { npu } \quad t \rightarrow \pm \infty
$$

ii) если, кроме того, $d=1$ и все функции $F_{k}\left(y_{k}\right)$ вещественно-аналитические на $\mathbb{R}$, то существуют такие стационарние состояния $S_{ \pm} \in \mathscr{S}$, зависящие от решения $Y(t)$, что

$$
Y(t) \stackrel{\mathscr{E}_{F}}{\longrightarrow} S_{ \pm} \quad \text { npu } \quad t \rightarrow \pm \infty
$$

Всюду ниже мы полагаем $d=1$, так как все доказательства распространяются без изменений на $d \geqslant 1$.

ЗАмЕчАния. i) Утверждение іi) этой теоремы вытекает из і) ввиду предложения 2.2.2.

ii) Из сходимости $(2.2 .2)$ и из $(2.1 .7),(0.1 .16)-(0.1 .17)$ по лемме Фату вытекает $(0.1 .23)$. 


\section{$\S$ 2.3. Существование динамики и априорные оценки}

При $N=1$ предложение 2.2 .2 доказано в $\S 2$ главы I. Для $N \geqslant 1$ доказательство аналогично. Решение строится методом Даламбера, т.е. при помоши представления (2.1.9). Однако при $N>1$ необходимо находить многократно отраженные волны от точек $x_{k}, k=1, \ldots, N$. Нелинейные уравнения для отраженных волн подробно рассмотрены в $\S 2$ главы I. Покажем,что из закона сохранения энергии $(E)$ вытекает следуюшая априорная оценка.

СлЕДСТВИЕ 2.3.1. Пусть выполнены условия (0.1.16)-(0.1.17). Тогда для любого решения $Y(t) \in C(\mathbb{R}, \mathscr{E})$ системь $(2.1 .8)$ все функции $y_{k}(t) \equiv u\left(x_{k}, t\right), t \in \mathbb{R}$, ограничены:

$$
\max _{1 \leqslant k \leqslant N}\left|y_{k}(t)\right| \leqslant B \quad \text { npu } \quad t \in \mathbb{R},
$$

где $B<\infty$ зависит от рассматриваемого решения.

На самом деле мы докажем более сильное утверждение. Положим $y_{k}=y_{k}(u)=$ $u\left(x_{k}\right)$ и $\bar{y}=\bar{y}(u)=\left(y_{1}, \ldots, y_{N}\right)$ для $u \in \mathscr{Q}$. Определим на $\mathscr{Q}$ функционал потенциальной энергии $\mathscr{U}:$ для $u \in \mathscr{Q}$

$$
\mathscr{U}(u) \equiv \mathscr{H}(u, 0)=\frac{1}{2} \int_{-\infty}^{\infty}\left|u^{\prime}(x)\right|^{2} d x+\sum_{k=1}^{N} V_{k}\left(u\left(x_{k}\right)\right) ; \quad y_{k} \equiv u\left(x_{k}\right)
$$

ЛЕмма 2.3.2. Пусть выпполнены условия (0.1.16)-(0.1.17). Тогда

$$
\mathscr{U}(u) \rightarrow \infty \quad n p u \quad|\bar{y}(u)| \rightarrow \infty .
$$

ДокАЗАТЕЛЬСТво. Покажем, что

$$
\sup _{\mathcal{U}(u) \leqslant E}|\bar{y}(u)|<\infty
$$

для любой ограниченной константы $E$. Действительно, из (2.3.2) и (0.1.17) вытекает

$$
\sup _{\mathcal{U}(u) \leqslant E} \int_{-\infty}^{\infty}\left|u^{\prime}(x)\right|^{2} d x=D<\infty .
$$

Поэтому из неравенства Коши-Буняковского получаем для всех $k, j$

$$
\sup _{\mathcal{U}(u) \leqslant E}\left|y_{k}-y_{j}\right|=\sup _{\mathcal{U}(u) \leqslant E}\left|\int_{x_{k}}^{x_{j}} u^{\prime}(x) d x\right| \leqslant\left|x_{k}-x_{j}\right|^{1 / 2} D^{1 / 2} .
$$

Отсюда и из (0.1.17) вытекает (2.3.4).

Из леммы 2.3.2 и (0.1.17) вытекает следствие 2.3.1. 
ЗАмЕчАниЕ. Из условий (0.1.16) вытекает, что гамильтониан $\mathscr{H}$ дифференцируем по Фреше на пространстве $\mathscr{E}$. Если $\left.u^{\prime \prime}\right|_{\Delta_{k}} \in L^{2}\left(\Delta_{k}\right) \forall k=1, \ldots, N+1$, то сушествуют пределы $u^{\prime}\left(x_{k} \pm 0\right) \forall k=1, \ldots, N$ и

$$
\begin{aligned}
\frac{\delta \mathscr{H}}{\delta v(x)} & =v(x), \\
\frac{\delta \mathscr{H}}{\delta u(x)} & =-u^{\prime \prime}(x)+\sum_{k=1}^{N}\left(-\left[u^{\prime}\left(x_{k}+0\right)-u^{\prime}\left(x_{k}-0\right)\right]+\nabla V_{k}\left(y_{k}\right)\right) \delta\left(x-x_{k}\right),
\end{aligned}
$$

где $y_{k} \equiv u\left(x_{k}\right)$. Поэтому (2.1.1) является формально гамильтоновой системой:

$$
\dot{u}=\frac{\delta \mathscr{H}}{\delta v}, \quad \dot{v}=-\frac{\delta \mathscr{H}}{\delta u} .
$$

Более точно, если мы допустим, что $\dot{v}(\cdot, t)$ - регулярное распределение, то из второго уравнения в (2.3.7) следует третье уравнение (2.1.1).

\section{$\S$ 2.4. Стационарные состояния}

Докажем предложение 2.2.2. Чтобы найти все стационарные решения, подставим $u(x, t)=s(x)$ в (2.1.1). Тогда из первого уравнения (2.1.1) вытекает, что $u^{\prime \prime}(x)=0$ для $x \in \mathbb{R} \backslash Q$, откуда

$$
s(x)=a_{k} x+b_{k} \quad \text { при } \quad x \in \Delta_{k}, \quad k=1, \ldots, N+1 .
$$

Из условия $s^{\prime} \in L^{2}(\mathbb{R})$ вытекает

$$
a_{1}=a_{N+1}=0 .
$$

Подставляя (2.4.1) во второе и третье уравнения (2.1.1), получаем для всех $k=1, \ldots, N$

$$
\left\{\begin{array}{l}
a_{k} x_{k}+b_{k}=a_{k+1} x_{k}+b_{k+1}\left(\equiv y_{k}\right), \\
0=F_{k}\left(y_{k}\right)+a_{k+1}-a_{k} .
\end{array}\right.
$$

Из (2.4.2) следует, что функция (2.4.1) определена однозначно своими значениями $y_{k}$ в точках $x_{k}, k=1, \ldots, N$ :

$$
a_{k}=\frac{y_{k}-y_{k-1}}{l_{k}}, \quad b_{k}=y_{k}-a_{k} x_{k}, \quad k=1, \ldots, N ; \quad b_{N+1}=y_{N} .
$$

Здесь $y_{0} \equiv y_{1}$ и $y_{N+1} \equiv y_{N}, l_{k}=x_{k}-x_{k-1}$ и $l_{1} \equiv l_{N+1} \equiv 1$ (например). Для неизвестных $y_{k}$ система (2.4.3) эквивалентна

$$
F_{k}\left(y_{k}\right)+\frac{y_{k+1}-y_{k}}{l_{k+1}}-\frac{y_{k}-y_{k-1}}{l_{k}}=0, \quad k=1, \ldots, N .
$$

Обозначим через $\mathscr{S}_{N}$ множество всех действительных решений $\left(y_{1}, \ldots, y_{N}\right) \in \mathbb{R}^{N}$ системы (2.4.5).

ЗАмечАниЕ. Поскольку $s(x) \in C^{2}\left(\bar{\Delta}_{k}\right) \forall k=1, \ldots, N+1$, то формулы (2.3.7) выполняются для стационарных состояний $(s(x), 0)$ системы (2.1.8). Следовательно, система (2.1.8) для стационарных состояний эквивалентна уравнению

$$
\frac{\delta \mathscr{U}}{\delta u}(s)=0 .
$$


ДоКАЗАТЕЛЬСТВо ПРЕДЛОЖЕНИЯ 2.2.2. Определим функцию на $\mathbb{R}^{N}$

$$
\mathscr{U}_{N}\left(y_{1}, \ldots, y_{N}\right)=\mathscr{U}(s)
$$

где $s=s(x)$ - функция (2.4.1) с $a_{k}$ и $b_{k}$, определенными согласно (2.4.4). Тогда из (2.3.2) вытекает, что

$$
\mathscr{U}_{N}\left(y_{1}, \ldots, y_{N}\right)=\sum_{k=1}^{N} V_{k}\left(y_{k}\right)+\frac{1}{2} \sum_{k=2}^{N}\left|\frac{y_{k}-y_{k-1}}{l_{k}}\right|^{2} l_{k} .
$$

Приведенное вьше замечание наводит на мысль, что система (2.4.5) эквивалентна

$$
\frac{\partial \mathscr{U}_{N}}{\partial y_{k}}\left(y_{1}, \ldots, y_{N}\right)=0, \quad k=1, \ldots, N
$$

Эта эквивалентность легко проверяется прямым вычислением.

ЗАмечание. Из (2.3.3) вытекает

$$
\mathscr{U}_{N}\left(y_{1}, \ldots, y_{N}\right) \rightarrow \infty \text { при }\left|\left(y_{1}, \ldots, y_{N}\right)\right| \rightarrow \infty
$$

Следовательно, $\mathscr{U}_{N}$ достигает минимального значения в некоторой точке $\left(y_{1}, \ldots, y_{N}\right)$ $\in \mathbb{R}^{N}$, так что (2.4.9) вьполняется и $\mathscr{S} \neq \varnothing$.

Возьмем $y_{0}(\lambda)=y_{1}(\lambda)=\lambda \in \mathbb{R}$. Тогда можно определить однозначно $y_{2}(\lambda), \ldots$, $y_{N}(\lambda), y_{N+1}(\lambda)$ по формулам (2.4.5) c $k=1, \ldots, N$. Следовательно, непрерьвное отображение $I: \mathscr{E}_{F} \rightarrow \mathbb{R}$, определенное формулой $I(u(x), v(x))=u\left(y_{1}\right)$, является инъективным на $\mathscr{S}$. Поэтому предложение 2.2 .2 вытекает из леммы 0.1 .4 и следуюшей леммы.

Лемма 2.4.1. $Z=I \mathscr{S}$ - дискретное множество в $\mathbb{R}$.

ДокАЗАТЕЛЬСТво. Все функции $y_{k}(\lambda)$ - действительно-аналитические на $\mathbb{R}, k=$ $2, \ldots, N+1$. Набор $\left\{y_{k}(\lambda): k=2, \ldots, N+1\right\}$ определяет стационарное решение $s_{\lambda}(x)$ вида $(2.4 .4),(2.4 .1)$ тогда и только тогда, когда $a_{N+1}=0$, т.е. $y_{N+1}=y_{N}$. Следовательно, мы получаем уравнение для $\lambda$

$$
y_{N+1}(\lambda)=y_{N}(\lambda) \text {. }
$$

В обеих частях этого равенства стоят вешественно-аналитические функции от $\lambda \in \mathbb{R}$. Следовательно, множество $Z$ всех решений уравнения (2.4.11) или является дискретным в $\mathbb{R}$, или $Z=\mathbb{R}$. Покажем, что случай $Z=\mathbb{R}$ невозможен в силу условий (0.1.16), (0.1.17), даже если функции $F_{k}$ не являются вещественно-аналитическими.

Лемма 2.4.2. Пусть $F_{k} \in C^{1}(\mathbb{R}) \quad \forall i u V_{k}$ удовлетворяют условиям (0.1.16), (0.1.17). Тогда $Z \neq \mathbb{R}$. 
ДокАЗАТЕльСтво. Предположим обратное: $Z=\mathbb{R}$. Тогда

$$
\mathscr{U}_{N}\left(y_{1}(\lambda), \ldots, y_{N}(\lambda)\right)=\text { const } \quad \text { при } \lambda \in \mathbb{R} .
$$

Действительно, поскольку $F_{k} \in C^{1}(\mathbb{R})$, то $y_{k}(\lambda) \in C^{1}(\mathbb{R})$ для всех $k=1, \ldots, N$. Поэтому из (2.4.9) вытекает

$$
\frac{d}{d \lambda} \mathscr{U}_{N}\left(y_{1}(\lambda), \ldots, y_{N}(\lambda)\right)=\sum_{k=1}^{N} \frac{\partial \mathscr{U}_{N}}{\partial y_{k}} \frac{d y_{k}}{d \lambda}(\lambda)=0, \quad \lambda \in \mathbb{R}
$$

С другой стороны, из (2.4.8) следует

$$
\mathscr{U}_{N}\left(y_{1}(\lambda), \ldots, y_{N}(\lambda)\right)=\sum_{k=1}^{N} V_{k}\left(y_{k}(\lambda)\right)+\frac{1}{2} \sum_{k=2}^{N}\left|\frac{y_{k}(\lambda)-y_{k-1}(\lambda)}{l_{k}}\right|^{2} l_{k}
$$

Следовательно, вторая сумма в правой части (2.4.14) ограничена при $\lambda \in \mathbb{R}$ ввиду (2.4.12) и (0.1.16). Поэтому

$$
y_{k}(\lambda) \rightarrow \infty \text { при } y_{1}=\lambda \rightarrow \infty, \quad \forall k=1, \ldots, N \text {. }
$$

Но тогда первая сумма в правой части (2.4.8) стремится к бесконечности при $\lambda \rightarrow \infty$ ввиду (0.1.17). Следовательно,

$$
\mathscr{U}_{N}\left(y_{1}(\lambda), \ldots, y_{N}(\lambda)\right) \rightarrow \infty \text { при } \lambda \rightarrow \infty
$$

что противоречит (2.4.12).

Итак, множество $Z$ дискретно в $\mathbb{R}$, и, следовательно, $\mathscr{S}_{N}$ дискретно в $\mathbb{R}^{N}$. Остается заметить, что условие $\mathscr{H}(s(x), 0) \leqslant h$ для стационарных состояний $(s(x), 0) \in \mathscr{E}$ системы $(2.1 .18)$ эквивалентно $\mathscr{U}_{N}\left(y_{1}, \ldots, y_{N}\right) \leqslant h$. Множество этих точек $\left(y_{1}, \ldots, y_{N}\right) \in$ $\mathbb{R}^{N}$ ограничено в $\mathbb{R}^{N}$ в силу (2.4.10). Следовательно, пересечение этого множества с дискретным множеством $\mathscr{S}_{N}$ конечно для любого $h \in \mathbb{R}$.

ЗАмечАниЕ. (2.4.9) следует из (2.4.6) и (2.4.5). Но обратная импликация не очевидна. При $x \in \mathbb{R} \backslash Q$ равенство (2.4.6) вытекает из (2.4.1) непосредственно. Грубо говоря, (2.4.9) обеспечивает (2.4.6) также и при $x \in Q$.

ПримеР 0. Пусть каждый потенциал $V_{k}(y)$ - полином четной степени $p_{k}+1 \geqslant 2$ с положительным старшим коэффициентом. Тогда все условия леммы 2.4.2 вьполнены. Все $F_{k}(y)$ являются полиномами степени $p_{k} \geqslant 1$. В силу $(2.4 .5)$ все функции $y_{k}(\lambda)$ являются полиномами степени $p_{1} \cdots p_{k-1}$. Следовательно, (2.4.11) имеет не более чем $\bar{p} \equiv p_{1} \cdots p_{N}$ корней $\lambda \in \mathbb{R}$, и множество $\mathscr{S}$ состоит не более, чем из $\bar{p}$ точек.

ЗАмЕчАнИЕ. Если потенциалы $V_{k}$ не удовлетворяют либо условию (0.1.16), либо (0.1.17), либо условию аналитичности, то множество $\mathscr{S}$ может не быть дискретньп. Это показывают следующие примеры. 
ПримеР 1. Отбросим условие (0.1.16): рассмотрим систему (2.1.1) при $N=2$, $x_{1}=-1, x_{2}=1$ и

$$
V_{k}(y)=-\frac{y^{2}}{2}, \quad k=1,2 .
$$

Тогда $F_{k}(y)=y-$ сила отталкивания от точки $y=0$. В этом случае система (2.1.1) имеет континуум решений (см. рис. 9)

$$
s_{\lambda}(x)=\left\{\begin{array}{cl}
\lambda, & x \leqslant-1, \\
-\lambda x, & -1 \leqslant x \leqslant 1, \\
-\lambda, & x \geqslant 1 .
\end{array}\right.
$$

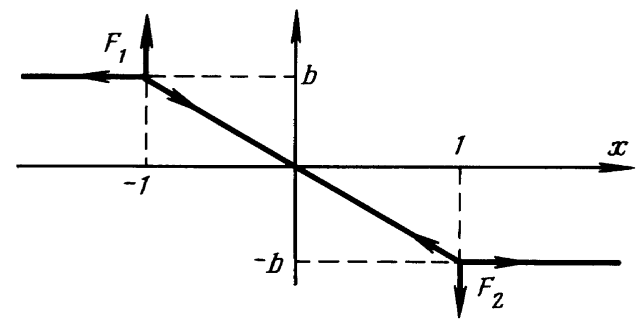

Рис. 9

Здесь $y_{1}=s_{\lambda}(-1)=\lambda$ - произвольное действительное число, т.е. $Y_{1}=\mathbb{R}$. Потенциалы $V_{k}(y)$ - вещественно-аналитические функции. Условие (0.1.17) не выполняется, но может быть формально обеспечено. А именно, добавим упругую силу $F_{3}(y)=-y$ с потенциалом $V_{3}(y)=y^{2} / 2$ в точке $x_{3}=0$. Тогда условие (0.1.17) вьполнено. Функции (2.4.18) остаются стационарными решениями новой системы, включающей три силы. Действительно, поскольку $s_{\lambda}(0)=0$ при всех $\lambda \in \mathbb{R}$, то сила $F_{3}$ равна нулю. Итак, здесь выполнены условие (0.1.17) и условие аналитичности, но множество $\mathscr{S}$ не является дискретным.

ПримеР 2. Отбросим условие (0.1.17): возьмем $V_{k}(y) \equiv$ const при всех $i$, тогда $F_{k}(y) \equiv 0$. Следовательно, $s_{\lambda}(x) \equiv \lambda$ при $x \in \mathbb{R}$ является стационарным решением системы (2.1.1). Значит, $Y_{1}=\mathbb{R}$, как в примере 1. Условие (0.1.16) и условие аналитичности выполнены, но множество $\mathscr{S}$ не является дискретным.

ПримеР 3. Отбросим условие аналитичности. Рассмотрим такие потеншиалы $V_{k}(y)$, что:

i) $V_{k}(y) \in C^{2}(\mathbb{R})$ удовлетворяют условию (0.1.16),

ii) $V_{k}(y) \rightarrow \infty$ при $|y| \rightarrow \infty$ для всех $k=1, \ldots, N$, и для некоторых констант $C_{k n} \in \mathbb{R}$

$$
V_{k}(y)=C_{k n} \text { при } y \in I_{n} \equiv[2 n, 2 n+1] \quad \forall n \in \mathbb{Z} \text {. }
$$


Ясно, что такие функции $V_{k}$ существуют и не являются вещественно-аналитическими. $F_{k}(y) \equiv 0$ при $y \in I_{n} \forall n \in \mathbb{Z}$. Поэтому функции $s_{\lambda}(x) \equiv \lambda, x \in \mathbb{R},-$ стационарные решения системы (2.1.1), если $\lambda \in I_{n}$ для некоторого $n \in \mathbb{Z}$. Значит, множество $\mathscr{S}$ не является дискретным, хотя условия (0.1.16) и (0.1.17) выполнены. Отметим однако, что здесь $Y_{1} \neq \mathbb{R}$ в соответствии с леммой 2.4.2.

\section{$\S$ 2.5. Долговременная асимптотика}

Мы доказываем здесь теорему 2.2.3.

Компактное притягивающее множество. Сначала построим компактное притягивающее множество $\mathscr{A}$ для рассматриваемой траектории $Y(t)$. Это множество состоит из кусочно-линейных функций. Для $a=\left\{\left(a_{k}, b_{k}\right) \in \mathbb{R}^{2}: k=1, \ldots, N+1\right\} \in$ $\left(\mathbb{R}^{2}\right)^{N+1}$ положим (ср. $\left.(2.4 .1)\right)$

$$
u_{a}(x)=a_{k} x+b_{k} \text { при } x \in \Delta_{k}, \quad k=1 \ldots, N+1,
$$

и пусть $A_{\mathscr{E}}=\left\{a \in\left(\mathbb{R}^{2}\right)^{N+1}: u_{a}\left(x_{k}-0\right)=u_{a}\left(x_{k}+0\right), k=1, \ldots, N ; a_{1}=a_{N+1}=0\right\}$. Тогда $\left(u_{a}(x), 0\right) \in \mathscr{E}$ для каждого $a \in A_{\mathscr{E}}$.

ОПРЕДЕЛЕНИЕ 2.5.1. $\mathscr{A}=\left\{S_{a}=\left(u_{a}(x), 0\right) \in \mathscr{E}: a \in A_{\mathscr{E}},\left|u\left(x_{k}\right)\right| \leqslant B\right.$ при всех $k=1, \ldots, N\}$, где $B$ - гранища $(2.3 .1)$.

Множество $\mathscr{A}$ компактно в $\mathscr{E}_{F}$, поскольку оно гомеоморфно компакту в $\left(\mathbb{R}^{2}\right)^{N+1}$. В следующем параграфе будет доказана

Лемма 2.5.2. Пусть все условия теоремы 2.2.3 виполнены. Тогда $Y(t) \stackrel{\mathscr{E}_{F}}{\longrightarrow} \mathscr{A}$ npu $t \rightarrow \pm \infty$.

ДОКАЗАТЕЛЬСТВо ТЕОРЕМЫ 2.2.3. Из леммы 2.5.2 следует, что орбита $O(Y)$ предкомпактна в $\mathscr{E}_{F}$. Поэтому из следующей леммы вытекает (2.2.1).

ЛЕмма 2.5.3. $\Omega(Y)$ содержится в $\mathscr{S}$.

ДокАЗАТЕльство. $\Omega(Y) \subset \mathscr{A}$, поскольку $\mathscr{A}$-притягиваюшее множество. Кроме того, множество $\Omega(Y)$ инвариантно относительно групшы $W_{t}, t \in \mathbb{R}$, в силу непрерывности $W_{t}$ в $\mathscr{E}_{F}$. Поэтому для каждого $\bar{Y} \in \Omega(Y)$ существует $C^{2}$-кривая $t \mapsto a(t) \in A_{\mathscr{E}}$, для которой $W_{t} \bar{Y}=S_{a(t)}$. Это значит, что $S_{a(t)}$ - решение системы (2.1.8). Но тогда $\dot{a}(t)=0$, т.е. $a(t) \equiv a$ и $\bar{Y}=S_{a} \in \mathscr{S}$.

\section{$\S$ 2.6. Притяжение к компактному множеству}

Мы доказьваем здесь лемму 2.5.2. Достаточно построить такую функцию $a(t) \in$ $C\left[0, \infty ; A_{\mathscr{E}}\right)$, что для любого $R>0($ cр. $(0.1 .14))$

$$
\left\|Y(t)-S_{a(t)}\right\|_{R} \rightarrow 0 \text { при } t \rightarrow+\infty
$$


Без ограничения обшности можно считать, что $x_{1}=0$. Тогда $S_{a}\left(x_{1}\right)=b_{1}$ и $(2.6 .1)$ принимает вид

$$
\int_{-R}^{R}\left|u^{\prime}(x, t)-u_{t}^{\prime}(x)\right|^{2} d x+\int_{-R}^{R}|\dot{u}(x, t)|^{2} d x+\left|y_{1}(t)-b_{1}(t)\right| \rightarrow 0 \text { при } t \rightarrow+\infty .
$$

Положим $b_{1}(t)=y_{1}(t)$ при $t>0$, тогда (2.6.2) в обозначениях (2.5.1) принимает вид

$$
\begin{aligned}
\int_{-R}^{x_{1}}\left|u^{\prime}(x, t)\right|^{2} d x & +\sum_{2 \leqslant k \leqslant N} \int_{x_{k-1}}^{x_{k}}\left|u^{\prime}(x, t)-a_{k}(t)\right|^{2} d x \\
& +\int_{x_{N}}^{R}\left|u^{\prime}(x, t)\right|^{2} d x+\int_{-R}^{R}|\dot{u}(x, t)|^{2} d x \rightarrow 0 \text { при } t \rightarrow+\infty,
\end{aligned}
$$

если $R>a$. Остается проверить эту сходимость с некоторьми $a_{k}(t)$.

ПРеДЛОЖЕНИЕ 2.6.1. Для любого $k=1, \ldots, N+1$ существует такая функция $c_{k}(t) \in C\left(\mathbb{R}_{+}\right)$, что для любого $R>0$

$$
\left\|f_{k}^{\prime}(t+\cdot)-c_{k}(t)\right\|_{R}+\| g_{k}^{\prime}(t+\cdot)+c_{k}\left(t \|_{R} \rightarrow 0 \quad \text { npu } \quad t \rightarrow+\infty\right.
$$

$u$, кроме того, $c_{1}(t)=c_{N+1}(t)=0$ при $t \in \mathbb{R}$.

Из этого предложения и преставления Даламбера (2.1.9) очевидно вытекает сходимость (2.6.3) с $a_{k}(t)=2 c_{k}(t)$.

2.6.1. Релаксация на бесконечности. Докажем предложение 2.6.1, используя подходящее понятие "релаксации", описьвающее долговременное поведение типа (2.6.4). Введем стандартную соболевскую метрику $\|\cdot\|_{R}$ пространства $H^{1}(-R, R)$ :

$$
\|y\|_{R}^{2} \equiv \mid y(s)\left\|_{R}^{2}+\right\| \dot{y}(s) \|_{R}^{2}
$$

ОПРЕДЕЛЕНИЕ 2.6.2. і) Функция $z(t) \in L_{\mathrm{loc}}^{2}\left(\mathbb{R}_{+}\right)$релаксирует в $L^{2}$, если сушествует такая функция $\bar{z}(t)$, что для любого $R>0$

$$
|z(t+\cdot)-\bar{z}(t)|_{R}^{2} \rightarrow 0 \text { при } t \rightarrow+\infty .
$$

Будем записывать это отношение в виде $z(t) \stackrel{L^{2}}{\sim} \bar{z}(t)$ при $t \rightarrow+\infty$.

ii) Функция $y(t) \in H_{\mathrm{loc}}^{1}\left(\mathbb{R}_{+}\right)$релаксирует в $H^{1}$, если сушествует функция $\bar{y}(t)$ такая, что для любого $R>0$

$$
\|y(t+\cdot)-\bar{y}(t)\|_{R} \rightarrow 0 \text { при } t \rightarrow+\infty .
$$

Будем записьвать это отношение в виде $y(t) \stackrel{H^{1}}{\sim} \bar{y}(t)$ при $t \rightarrow+\infty$.

Следуюшие свойства релаксации очевидны.

Ro. В (2.6.7) без ограничения обшности можно положить $\bar{y}(t) \equiv y(t)$. 
R1. Если функция $z(t)$ релаксирует в $H^{1}$, то она также релаксирует и в $L^{2}$.

R2. Чтобы функция $z(t)$ релаксировала в $L^{2}$, достаточно, чтобы

$$
\int_{0}^{\infty}|z(t)|^{2} d t<\infty
$$

При этом можно положить $\bar{z}(t) \equiv 0$, т.е. $z(t) \stackrel{L^{2}}{\sim} 0$ при $t \rightarrow+\infty$.

R3. Чтобы функция $y(t)$ была релаксируюшей в $H^{1}$, достаточно потребовать

$$
\int_{0}^{\infty}|\dot{y}(t)|^{2} d t<\infty
$$

Действительно, тогда по неравенству Коши-Буняковского для $|s| \leqslant R$

$$
|y(t+s)-y(t)|=\left|\int_{t}^{t+s} \dot{y}(\tau) d \tau\right| \leqslant R^{1 / 2}\left|\dot{y}^{2}(t+\cdot)\right|_{R}^{1 / 2} \rightarrow 0 \text { при } t \rightarrow+\infty .
$$

R4. Если $y(t)$ релаксирует в $H^{1}$, то ее производная $z(t) \equiv \dot{y}(t)$ релаксирует в $L^{2}$ и $\dot{y}(t) \stackrel{L^{2}}{\sim} 0$ при $t \rightarrow+\infty$ в силу $\mathbf{R 2 .}$

R5. Если $z(t)$ релаксирует в $L^{2}$, то интеграл $y(t)=\int_{t+h_{-}}^{t+h_{+}} z(s) d s$ релаксирует в $H^{1}$ для любого $h_{ \pm} \in \mathbb{R}$. При этом можно взять

$$
\bar{y}(t) \equiv\left(h_{+}-h_{-}\right) \bar{z}(t)
$$

R6. Если $y(t) \sim \bar{y}(t)$ при $t \rightarrow+\infty$ в $H^{1}$ (или в $L^{2}$ ), то $y(t+h) \sim \bar{y}(t)$ в $H^{1}$ (или в $\left.L^{2}\right)$ для любого $h \in \mathbb{R}$.

R7. Множество всех функций $z(t)$, релаксируюших в $L^{2}$ (или в $H^{1}$ ), является линейньмм пространством, и $z_{1}(t)+z_{2}(t) \sim \bar{z}_{1}(t)+\bar{z}_{2}(t)$, если $z_{j}(t) \sim \bar{z}_{j}(t), j=1,2$.

R8. Пусть $F(\cdot) \in C^{1}(\mathbb{R})$ и $y(t) \in C_{b}\left(\mathbb{R}_{+}\right)$. Тогда из $y(t) \stackrel{L^{2}}{\sim} \bar{y}(t)$ вытекает, что $F(y(t)) \stackrel{L^{2}}{\sim} F(\bar{y}(t))$.

В следуюшем параграфе мы докажем релаксацию данных Коши решения $u(x, t)$ на прямых $x=x_{k} \pm 0$,

$$
y_{k}(t) \equiv u\left(x_{k}, t\right) \quad \text { и } \quad z_{k}^{ \pm}(t) \equiv u^{\prime}\left(x_{k} \pm 0, t\right), \quad t \in \mathbb{R}, \quad k=1, \ldots, N .
$$

Лемма 2.6.3. Все функции $y_{k}(t), k=1, \ldots, N$, релаксируют в $H^{1}$, и все функиии $z_{k}^{ \pm}(t), k=1, \ldots, N$, релаксируют в $L^{2}$. При этом $y_{1}, y_{N+1} \stackrel{H^{1}}{\sim} 0 u z_{1}, z_{N+1} \stackrel{L^{2}}{\sim} 0$ npu $t \rightarrow+\infty$.

Эта лемма, представление Даламбера (2.1.9) и свойства $\mathbf{R 0}-\mathbf{R 8}$ релаксации позволяют доказать предложение 2.6.1. 
ДОКАЗАТЕЛЬСТВО ПРЕДЛОЖЕНИЯ 2.6.1. Докажем (2.6.4) при $k \geqslant 2$ (случай $k=1$ совершенно аналогичен). Представление (2.1.9) приводит к хорошо известной формуле Даламбера: при $x_{k}<x<x_{k+1}$

$$
u(x, t)=\frac{y_{k}\left(t-\left(x-x_{k}\right)\right)+y_{k}\left(t+\left(x-x_{k}\right)\right)}{2}+\frac{1}{2} \int_{t-\left(x-x_{k}\right)}^{t+\left(x-x_{k}\right)} z_{k}^{+}(s) d s .
$$

Следовательно,

$$
\begin{aligned}
\dot{u}(x, t) & =\frac{\dot{y}_{k}\left(t-\left(x-x_{k}\right)\right)+\dot{y}_{k}\left(t+\left(x-x_{k}\right)\right)}{2}+\frac{z_{k}^{+}\left(t+\left(x-x_{k}\right)\right)-z_{k}^{+}\left(t-\left(x-x_{k}\right)\right)}{2}, \\
u^{\prime}(x, t) & =\frac{-\dot{y}_{k}\left(t-\left(x-x_{k}\right)\right)+\dot{y}_{k}\left(t+\left(x-x_{k}\right)\right)}{2}+\frac{z_{k}^{+}\left(t+\left(x-x_{k}\right)\right)+z_{k}^{+}\left(t-\left(x-x_{k}\right)\right)}{2} .
\end{aligned}
$$

Поэтому из леммы 2.6.3 и R7, R6, R4 вытекает $(2.6 .4)$ с $c_{k}(t)=-\overline{z_{k}^{+}}(t) / 2$.

ЗАмЕчАниЕ. (2.6.3) можно получить непосредственно из (2.6.14) и леммы 2.6.3. Мы выводим (2.6.3) из предложения 2.6.1 для простоты изложения.

Остается доказать лемму 2.6.3. Вьведем ее, анализируя рассеяние энергии в бесконечность.

\subsection{2. Рассеяние энергии в бесконечность.}

Лемма 2.6.4. Справедлива оценка

$$
\int_{0}^{\infty}\left(\left|\dot{y}_{1}(t)\right|^{2}+\left|z_{1}^{-}(t)\right|^{2}+\left|\dot{y}_{N+1}(t)\right|^{2}+\left|z_{N+1}^{+}(t)\right|^{2}\right) d t<\infty .
$$

ДокАЗАТЕЛЬСТво. Из представлений Даламбера (2.1.9) при $k=1$ и $k=N+1$ вытекает, что (2.6.15) эквивалентно оценке

$$
\int_{0}^{\infty}\left(\left|f_{1}^{\prime}\left(t-x_{1}\right)\right|^{2}+\left|g_{1}^{\prime}\left(t+x_{1}\right)\right|^{2}+\left|f_{N+1}^{\prime}\left(t-x_{N}\right)\right|^{2}+\left|g_{N+1}^{\prime}\left(t+x_{N}\right)\right|^{2}\right) d t<\infty
$$

Здесь интегралы от $f_{1}^{\prime}, g_{N+1}^{\prime}$ ограничены ввиду формул Даламбера

$$
\begin{gathered}
f_{1}(-x)=\frac{u^{0}(x)}{2}-\frac{1}{2} \int_{x_{1}}^{x} v^{0}(s) d s \text { при }-x<x_{1}, \\
g_{N+1}(x)=\frac{u^{0}(x)}{2}+\frac{1}{2} \int_{x_{1}}^{x} v^{0}(s) d s \text { при } x>x_{N},
\end{gathered}
$$

поскольку $\left(u^{0}, v^{0}\right) \in \mathscr{E}$. Чтобы доказать (2.6.16) для $g_{1}^{\prime}, f_{N+1}^{\prime}$, введем для $Y=$ $(u(x), v(x)) \in \mathscr{E}$ функционал энергии на отрезке $\Delta=\left[x_{1}, x_{N}\right]$ :

$$
\mathscr{H}_{\Delta}(Y)=\frac{1}{2} \int_{x_{1}}^{x_{N}}\left[|v(x)|^{2}+\left|u^{\prime}(x)\right|^{2}\right] d x+\sum_{k=1}^{N} V_{k}\left(y_{k}\right), \quad \text { где } y_{k}=u\left(x_{k}\right) .
$$


Рассмотрим поток энергии, исходяший из $\Delta$ : из (2.1.1) и (2.1.9) с $k=1$ и $k=N+1$ вытекает (для кусочно-гладких начальных данных $\left(u^{0}, v^{0}\right)$ )

$$
\begin{aligned}
\frac{d}{d t} \mathscr{H}_{\Delta}(Y(t))=\left.\dot{u} u^{\prime}\right|_{x=x_{1}-0} ^{x=x_{N}+0}=\left|f_{1}^{\prime}\left(t-x_{1}\right)\right|^{2}-\left|g_{1}^{\prime}\left(t+x_{1}\right)\right|^{2} \\
\quad+\left|g_{N+1}^{\prime}\left(t+x_{N}\right)\right|^{2}-\left|f_{N+1}^{\prime}\left(t-x_{N}\right)\right|^{2} \text { при п.в. } t \in \mathbb{R} .
\end{aligned}
$$

Интегрируя, получаем энергетическое тождество,

$$
\begin{aligned}
\mathscr{H}_{\Delta} & (Y(t))+\int_{0}^{t}\left(\left|g_{1}^{\prime}\left(s+x_{1}\right)\right|^{2}+\left|f_{N+1}^{\prime}\left(s-x_{N}\right)\right|^{2}\right) d s \\
& =\mathscr{H}_{\Delta}(Y(0))+\int_{0}^{t}\left(\left|f_{1}^{\prime}\left(s-x_{1}\right)\right|^{2}+\left|g_{N+1}^{\prime}\left(s+x_{N}\right)\right|^{2}\right) d s \text { при } t \in \mathbb{R} .
\end{aligned}
$$

Поэтому (2.6.16) для $g_{1}^{\prime}, f_{N+1}^{\prime}$ следует из (2.6.16) для $f_{1}^{\prime}, g_{N+1}^{\prime}$, поскольку

$$
\inf _{Y \in \mathscr{E}} \mathscr{H}_{\Delta}(Y)>-\infty
$$

в силу (0.1.16).

2.6.3. Лемма о релаксации. Мы доказьваем здесь лемму 2.6 .3 индукцией по $k$.

$k=1$ и $k=N+1$. Из (2.6.15) вытекает релаксация $y_{1}(t), y_{N+1}(t)$ и $z_{1}^{-}(t), z_{N+1}^{+}(t)$ в силу $\mathbf{R 3}$ и $\mathbf{R 2}$ соответственно. Следовательно, релаксация $z_{1}^{+}(t), z_{N+1}^{-}(t)$ вытекает из $\mathbf{R 7}$ и $\mathbf{R 8},(2.3 .1)$ в силу третьего уравнения $(2.1 .1)$ с $k=1, N$, т.е.

$$
z_{k}^{+}(t)-z_{k}^{-}(t)=-F_{k}\left(y_{k}(t)\right), \quad t \in \mathbb{R}
$$

$k=2$. Докажем релаксацию $y_{2}(t)$ и $z_{2}^{-}(t)$. Во-первых, из (2.6.13) с $k=2$ вытекает $(2.6 .20)$

$$
y_{2}(t)=u\left(x_{2}, t\right)=\frac{y_{1}\left(t-l_{2}\right)+y_{1}\left(t+l_{2}\right)}{2}+\frac{1}{2} \int_{t-l_{2}}^{t+l_{2}} z_{1}^{+}(s) d s, \text { где } l_{2} \equiv\left|x_{2}-x_{1}\right| .
$$

Поэтому из $\mathbf{R 5}$ и $\mathbf{R 6}$ следует релаксация $y_{2}(t)$ в $H^{1}$. Во-вторых, продифференцируем (2.6.13) и получим

(2.6.21) $z_{2}^{-}(t) \equiv u^{\prime}\left(x_{2}-0, t\right)=\frac{-\dot{y}_{1}\left(t-l_{2}\right)+\dot{y}_{1}\left(t+l_{2}\right)}{2}+\frac{z_{1}^{+}\left(t+l_{2}\right)+z_{1}^{+}\left(t-l_{2}\right)}{2}$.

Поэтому из $\mathbf{R 2}, \mathbf{R} 6$ и $\mathbf{R 7}$ вытекает релаксация $z_{2}^{-}(t)$ в $L^{2}$. Доказательство леммы 2.6.3 может быть завершено по индукции. 
Глава III. Нелинейная струна

\section{с пространственно-локализованной нелинейностью}

Устанавливается сходимость к стационарным состояниям для одномерного нелинейного волнового уравнения с нелинейным членом, сосредоточенным на ограниченном отрезке прямой. Результат обобщает [33].

\section{§3.1. Введение и основные результаты}

Мы установливаем сходимость типа $(0.1 .11),(0.1 .12)$ к стационарным состояниям для решений задачи Коши

$$
\begin{gathered}
\ddot{u}(x, t)=u^{\prime \prime}(x, t)+f(x, u(x, t)), \quad x \in \mathbb{R}, \quad t \in \mathbb{R}, \\
\left.u\right|_{t=0}=u^{0}(x),\left.\quad \dot{u}\right|_{t=0}=v^{0}(x) .
\end{gathered}
$$

Решения $u(x, t)$ принимают значения в $\mathbb{R}^{d} \mathrm{c} d \geqslant 1$. Мы предполагаем, что $f(x, u)=0$ для $|x| \geqslant a$ при некотором $a>0$. Физически, уравнение (3.1.1) описьвает малые поперечные колебания струны, взаимодействующей с упругой нелинейной средой вдоль отрезка $[-a, a]$. Мы используем конфигурационное пространство $\mathscr{Q}$ и фазовые пространства $\mathscr{E}, \mathscr{E}_{F}$, введенные в определении 2.1.1. Мы рассматриваем общие функции $f(x, u)$, удовлетворяюшие предположениям (0.1.18)-(0.1.20), и через $V(x, u)=$ $\chi(x) V(u)$ обозначаем потенциал нелинейной силы. При этих предположениях уравнение (3.1.1) формально является гамильтоновой системой с фазовым пространством $\mathscr{E}$ и с гамильтонианом

$$
\mathscr{H}(u, v)=\int_{\mathbb{R}}\left[\frac{1}{2}|v(x)|^{2}+\frac{1}{2}\left|u^{\prime}(x)\right|^{2}+V(x, u(x))\right] d x \quad \text { для }(u, v) \in \mathscr{E} .
$$

Мы рассматриваем решения $u(x, t)$, для которых $Y(t)=(u(\cdot, t), \dot{u}(\cdot, t)) \in C(\mathbb{R}, \mathscr{E})$, и записьваем задачу Коши (3.1.1)-(3.1.2) в виде

$$
\dot{Y}(t)=\mathscr{V}(Y(t)) \text { при } t \in \mathbb{R}, \quad Y(0)=Y^{0},
$$

где $Y^{0}=\left(u^{0}, v^{0}\right)$.

ПРЕДЛОЖЕНИЕ 3.1.1. Пусть $d \geqslant 1$ и выполняются условия (0.1.18)-(0.1.20). Тогда

i) для любого $Y^{0} \in \mathscr{E}$ задача Коши (3.1.4) имеет единственное решение $Y(t) \in C(\mathbb{R}, \mathscr{E})$

ii) отобрахсение $W_{t}: Y^{0} \mapsto Y(t)$ непрерывно в $\mathscr{E}$ и $\mathscr{E}_{F}$ для всех $t \in \mathbb{R}$;

iii) выполняется закон сохранения әнергии $(E)$.

Обозначим через $\mathscr{S}$ множество стационарных состояний $S=(s(x), 0) \in \mathscr{E}$ системы (3.1.4). Положим $\mathscr{S}^{h}=\{S \in \mathscr{S}: \mathscr{H}(S) \leqslant h\}$ для $h \in \mathbb{R}$. $\mathscr{S}^{h}$ является замкнутьм ограниченным множеством в $\mathscr{E}$ ввиду $(0.1 .18)-(0.1 .20)$ и (3.1.3):

$$
\sup _{S \in \mathscr{S} h}\|S\|_{\mathscr{E}}<\infty \quad \forall h \in \mathbb{R} .
$$


ПРЕДЛОЖЕНИЕ 3.1.2. Пусть въполняются условия (0.1.18)-(0.1.20) и, кроме того, $d=1$ и функиия $F(u)$ вещественно-аналитическая на $\mathbb{R}$. Тогда $\mathscr{S}^{h}-$ конечное множество для любого $h \in \mathbb{R}$.

Главный результат этой главы означает, что множество $\mathscr{S}$ является точечным аттрактором системы (3.1.4) в топологии Фреше пространства $\mathscr{E}_{F}$.

ТЕОРема 3.1.3. Пусть выполняются условия предложсения 3.1 .1 и начальное состояние $Y^{0} \in \mathscr{E}$. Тогда справедливы следующие утвержсдния.

i) Для решения $Y(t) \in C(\mathbb{R}, \mathscr{E})$ задачи Коши (3.1.4) орбита $O(Y)$ предкомпактна в $в \mathscr{E}_{F}$ u

$$
Y(t) \stackrel{\mathscr{E}_{F}}{\longrightarrow} \mathscr{S} \quad \text { npu } \quad t \rightarrow \pm \infty
$$

ii) Пусть, кроме того, $d=1$ и функиия $F(u)$ вещественно-аналитическая на $\mathbb{R}$. Тогда существуют такие стационарнье состояния $S_{ \pm} \in \mathscr{S}$, зависящие от решения $Y(t)$, что

$$
Y(t) \stackrel{\mathscr{E}_{F}}{\longrightarrow} S_{ \pm} \quad \text { npu } \quad t \rightarrow \pm \infty
$$

ЗАмЕчАнИЯ. і) Из сходимости (3.1.7) и (0.1.18)-(0.1.20), (3.1.3) по лемме Фату вытекает (0.1.23).

ii) Мы предполагаем для простоты изложения, что $f(x, u)=\chi(x) F(u)$. Все результаты этой главы могут быть легко перенесены на нелинейные члены $f(x, u)$ без такой структуры при подходяшем обобшении условий (0.1.18)-(0.1.20).

\section{$\S$ 3.2. Существование динамики и априорные оценки}

Выведем предложение 3.1.1 из принципа сжимающих отображений. Пусть $W_{t}^{0}$ обозначает динамическую группу, соответствуюшую линейному уравнению (3.1.1) c $f(x, u) \equiv 0$. Тогда задача Коши (3.1.4) для $Y(t) \in C(\mathbb{R}, \mathscr{E})$ эквивалентна интегральному уравнению

$$
Y(t)=W_{t}^{0} Y^{0}+\int_{0}^{t} W_{t-\tau}^{0}(0, f(\cdot, u(\cdot, \tau))) d \tau
$$

Следовательно, по принципу сжимающих отображений при некотором $\varepsilon>0$ сушествует единственное локальное решение $Y(t) \in C(-\varepsilon, \varepsilon ; \mathscr{E})$. Непрерывность $W_{t}$ в $\mathscr{E}$ и $\mathscr{E} F$ для малых $|t|$ вытекает из этого построения в силу соответствующих свойств операторов $W_{t}^{0}$.

Чтобы доказать сохранение энергии, предположим сначала, что $u^{0}(x) \in C^{2}(\mathbb{R})$, $v^{0}(x) \in C^{1}(\mathbb{R})$ и

$$
u^{0}(x)=v^{0}(x)=0 \text { при }|x| \geqslant R^{0} .
$$


Тогда из интегрального представления (3.2.1) следует, что $u(x, t) \in C^{2}(\mathbb{R} \times(-\varepsilon, \varepsilon))$ и

$$
u(x, t)=0 \text { при }|x| \geqslant \bar{R}+|t|, \quad \bar{R}=\max \left(R^{0}, a\right) .
$$

Поэтому сохранение энергии $(E)$ получается стандартным интегрированием по частям. Теперь сохранение энергии для произвольных $\left(u^{0}, v^{0}\right) \in \mathscr{E}$ вытекает из стандартных соображений плотности и непрерьвности.

Сохранение энергии $(E)$ и существование локального решения приводит к существованию глобального решения $Y(t) \in C(\mathbb{R}, \mathscr{E})$ со всеми указанными свойствами при любых $t \in \mathbb{R}$.

Однако, нам нужна более тонкая характеризация свойств решений.

ПРЕДЛОЖЕНИЕ 3.2.1. Пусть выполняются условия (0.1.18)-(0.1.20). Тогда

i) отображсние $W_{t}: Y^{0} \mapsto Y(t)$ непрерьвно по Липшицу в $\mathscr{E}_{F}$, т.е. для любъх $R, T>0$

$$
\left\|W_{t} Y_{1}-W_{t} Y_{2}\right\|_{R} \leqslant L_{T}\left\|Y_{1}-Y_{2}\right\|_{R+T} \quad \text { npu } \quad|t| \leqslant T,
$$

где константа $L_{T}$ ограничена при ограниченньх нормах $\left\|Y_{1}\right\|_{R+T},\left\|Y_{2}\right\|_{R+T}$;

ii) справедлива априорная оченка

$$
|u(x, t)| \leqslant \alpha+\beta \sqrt{|x|} \quad \text { npu } \quad x \in \mathbb{R}, \quad t \in \mathbb{R},
$$

где константы $\alpha$ и $\beta$ ограничень при ограниченной энергии $\mathscr{H}\left(Y^{0}\right)$;

iii) $u(x, \cdot) \in C\left(\mathbb{R}, H_{\text {loc }}^{1}(\mathbb{R})\right) u u^{\prime}(x, \cdot) \in C\left(\mathbb{R}, L_{\text {loc }}^{2}(\mathbb{R})\right)$;

iv) $\partial$ я п.в. $x \in \mathbb{R}$

$$
\int_{t}^{t+1}\left(|\dot{u}(x, s)|^{2}+\left|u^{\prime}(x, s)\right|^{2}+|u(x, s)|^{2}\right) d s \leqslant e(x)<\infty \quad n p u \quad t \in \mathbb{R}
$$

где е $(x)$ может зависеть от $x$ и от $\mathscr{H}\left(Y^{0}\right)$ и не зависит от $t \in \mathbb{R}$.

ДоказАтельство. і) Липшицева непрерьвность (3.2.4) для малых $T>0$ вытекает из построения $W_{t}$ методом сжимающих отображений в силу соответствующих свойств отображения $W_{t}^{0}$. Обобщение на произвольные $T>0$ очевидно.

ii) Из сохранения энергии $(E)$ и $(0.1 .19),(0.1 .20)$ вытекает

$$
D=\sup _{t \in \mathbb{R}} \int\left|u^{\prime}(x, t)\right|^{2} d x<\infty,
$$

причем $D$ ограничено при ограниченной энергии $\mathscr{H}\left(Y^{0}\right)$. Следовательно, по неравенству Коши-Буняковского

$$
\left|u(x, t)-u\left(x_{0}, t\right)\right|=\left|\int_{x_{0}}^{x} u^{\prime}(y, t) d y\right| \leqslant \sqrt{D} \sqrt{\left|x-x_{0}\right|} \text { при } x, x_{0}, t \in \mathbb{R} .
$$


Возьмем такое $x_{0}$, что $\chi\left(x_{0}\right)>0$. Тогда из $(E)$ и $(0.1 .19),(0.1 .20)$ следует

$$
\sup _{t \in \mathbb{R}}\left|u\left(x_{0}, t\right)\right|<\infty .
$$

Поэтому из (3.2.8) вытекает (3.2.5).

iii) Воспользуемся интегральным представлением (3.2.1). Первое слагаемое в правой части (3.2.1), очевидно, обладает нужными свойствами, а для интеграла это верно, поскольку $u(x, t) \in C\left(\mathbb{R}^{2}\right)$.

iv) Оценка (3.2.6) вытекает из $(E),(3.2 .5)$ и интегрального представления типа (3.2.1),

$$
Y(s)=W_{s-t}^{0} Y(t)+\int_{0}^{s-t} W_{\theta}^{0}(0, f(\cdot, u(\cdot, t+\theta))) d \theta
$$

справедливого в силу единственности решения. А именно, оценки типа (3.2.6) справедливы для первого слагаемого в правой части (3.2.9) в силу равномерной по $t$ оценки $(E)$ для $Y(t)$. Для второго слагаемого то же верно ввиду равномерных по $t$ оценок $(3.2 .5)$.

ЗАмЕчАниЕ. Из условий (0.1.18)-(0.1.20) вытекает, что гамильтониан $\mathscr{H}$ дифференцируем по Фреше на $\mathscr{E}$ и

$$
\frac{\delta \mathscr{H}}{\delta v(x)}=v(x), \quad \frac{\delta \mathscr{H}}{\delta u(x)}=-u^{\prime \prime}(x)-f(x, u(x)) .
$$

Поэтому уравнение (3.1.1) можно переписать в гамильтоновой форме:

$$
\dot{u}=\frac{\delta \mathscr{H}}{\delta v}, \quad \dot{v}=-\frac{\delta \mathscr{H}}{\delta u} .
$$

\section{§3.3. Стационарные состояния}

Докажем предложение 3.1.2. Чтобы найти все стационарные решения, подставим $u(x, t)=s(x)$ в (3.1.1). Тогда из (0.1.18)-(0.1.20) получаем

$$
\begin{cases}s^{\prime \prime}(x)+f(x, s(x))=0 & \text { при } x \in[-a, a], \\ s(x)=s( \pm a) & \text { при } \pm x \geqslant a,\end{cases}
$$

поскольку $s^{\prime}(x) \in L^{2}(\mathbb{R})$. Следовательно, непрерьвное отображение $I: \mathscr{E}_{F} \rightarrow \mathbb{R}$, определенное формулой $I(u(x), v(x))=u(-a)$, является инъективным на $\mathscr{S}$. Поэтому предложение 3.1.2 вытекает из следующей леммы.

ЛЕмма 3.3.1. $Z^{h}=I \mathscr{S}^{h}-$ конечное множество для каждого $h \in \mathbb{R}$. 
ДокаЗАТельство. Множество $\mathscr{S}^{h}$ компактно в $\mathscr{E}_{F}$ в силу (3.1.5) и (3.3.1). Следовательно, $\mathscr{Z}^{h}$ - замкнутое ограниченное подмножество в $\mathbb{R}$. Остается проверить, что $\mathscr{Z}^{h}$ не имеет предельных точек. Предположим противное, т.е. сушествует последовательность

$$
z_{k} \in Z^{h} \text { и } z_{k} \rightarrow \bar{z} \in Z^{h} \text { при } k \rightarrow \infty .
$$

Обозначим через $s_{\lambda}(x)$ решение задачи

$$
\left\{\begin{array}{l}
s_{\lambda}^{\prime \prime}(x)+f\left(x, s_{\lambda}(x)\right)=0 \text { при } x \in[-a, a] \\
s_{\lambda}^{\prime}(-a)=0, s_{\lambda}(-a)=\lambda
\end{array}\right.
$$

если это решение сушествует. Обозначим через $\Lambda$ множество всех $\lambda \in \mathbb{R}$, для которых решение $s_{\lambda}(x)$ сушествует. Продолжим $s_{\lambda}(x)$ на $|x|>a$ константами,

$$
s_{\lambda}=s_{\lambda}( \pm a) \text { при } \quad \pm x>a .
$$

Тогда $S_{\lambda}=\left(s_{\lambda}(x), 0\right) \in \mathscr{E}$ для любого $\lambda \in \Lambda$. Рассмотрим отображение $T: \Lambda \rightarrow \mathbb{R}$, определенное формулой

$$
T: \lambda \mapsto s_{\lambda}^{\prime}(a-0)
$$

Тогда $Z^{h}=\left\{\lambda \in \Lambda: T(\lambda)=0, \mathscr{H}\left(\left(s_{\lambda}(x), 0\right)\right) \leqslant h\right\}$. Множество $\Lambda$ является открытым, следовательно

$$
\Lambda=\bigcup_{1}^{\infty} \Lambda_{j}, \quad \Lambda_{j}=\left(\lambda_{j}^{-}, \lambda_{j}^{+}\right) \neq \varnothing, \quad \lambda_{j}^{-}, \lambda_{j}^{+} \notin \Lambda .
$$

Разумеется, $\bar{z} \in \Lambda_{l}$ при некотором $l$. Покажем, что

$$
\left|\lambda_{l}^{ \pm}\right|<\infty \quad \text { и } \lambda_{l}^{ \pm} \in \Lambda .
$$

Это противоречит (3.3.6) и завершает доказательство леммы 3.3.1.

Отображение $T: \Lambda \rightarrow \mathbb{R}$ вещественно-аналитическое, и $T(z)=0$ при $z \in Z$. Поэтому $T(\lambda)=0$ для всех $\lambda \in \Lambda_{l}$ в силу (3.3.2), т.е.

$$
\left(s_{\lambda}(x), 0\right) \in \mathscr{S} \quad \forall \lambda \in \Lambda_{l} .
$$

Обозначим через $\mathscr{U}$ функционал потенциальной энергии на конфигурационном пространстве $\mathscr{Q}$,

$$
\mathscr{U}(u) \equiv \mathscr{H}(u, 0)=\int_{-\infty}^{\infty}\left(\frac{1}{2}\left|u^{\prime}(x)\right|^{2}+V(x, u(x))\right) d x \text { при } u \in \mathscr{Q} .
$$


Тогда (3.3.1) эквивалентно равенству (вытекающему также из (3.2.11))

$$
\delta \mathscr{U}(s)=0,
$$

где $\delta \mathscr{U}$ - дифференциал $\Phi$ реше функционала $\mathscr{U}$ на пространстве $\mathscr{Q}$. Поэтому в силу (3.3.8)

$$
\frac{d}{d \lambda} \mathscr{U}\left(s_{\lambda}\right)=\left\langle\delta \mathscr{U}\left(s_{\lambda}\right), \frac{d}{d \lambda} s_{\lambda}\right\rangle=0 \text { при } \lambda \in \Lambda_{l},
$$

и, следовательно, функция $\lambda \mapsto \mathscr{U}\left(s_{\lambda}\right)$ постоянна на $\Lambda_{l}$. Отсюда аналогично $(3.2 .5)$ вытекает априорная оценка

$$
\left|s_{\lambda}(x)\right| \leqslant \alpha_{1}+\beta_{1} \sqrt{a} \text { при }|x| \leqslant a \text { и } \lambda \in \Lambda_{l}
$$

с некоторыми $\alpha_{1}$ и $\beta_{1}$, не зависяшими от $\lambda \in \Lambda_{l}$. Но тогда интервал $\Lambda_{l}$ ограничен, так как $s_{\lambda}(-a)=\lambda$. С другой стороны, из равномерных оценок (3.3.12) и уравнения (3.3.3) следует, что множество функций $\left\{s_{\lambda}(\cdot): \lambda \in \Lambda_{l}\right\}$ предкомпактно в $\mathscr{Q}$, откуда $\lambda_{l}^{ \pm} \in \Lambda_{l}$.

Предложение 3.1.2 доказано.

\section{$\S$ 3.4. Долговременная асимптотика}

Мы доказываем здесь теорему 3.1.3.

3.4.1. Компактное притягиваюшее множество. Построим компактное притягивающее множество $\mathscr{A}$ для рассматриваемой траектории $Y(t)$. Обозначим через $\bar{\alpha}, \bar{\beta}$ некоторые положительные константы, которые будут выбраны позднее.

ОПРЕДЕЛЕНИЕ 3.4.1. $\mathscr{A}=\mathscr{A}_{\bar{\alpha} \bar{\beta}}=\left\{S_{\lambda}=\left(s_{\lambda}(x), 0\right) \in \mathscr{E}: \lambda \in \Lambda_{\text {и }}\left|s_{\lambda}(x)\right| \leqslant \bar{\alpha}+\right.$ $\bar{\beta} \sqrt{x}$ при $|x| \leqslant a\}$.

Множество $\mathscr{A}$ компактно в $\mathscr{E}_{F}$ в силу уравнения (3.3.1). В следуюшем параграфе мы докажем лемму о притяжении:

Лемма 3.4.2. Пусть выполняются условия теоремы 3.1.3. Тогда

$$
Y(t) \stackrel{\mathscr{E}_{F}}{\longrightarrow} \mathscr{A}=\mathscr{A}_{\bar{\alpha} \bar{\beta}} \quad \text { npu } \quad t \rightarrow \pm \infty,
$$

если константы $\bar{\alpha}$ и $\bar{\beta}$ достаточно велики.

3.4.2. Доказательство теоремы 3.1.3. і) Из леммы 3.4 .2 следует, что орбита $O(Y)$ предкомпактна в $\mathscr{E}_{F}$. Поэтому из следуюшей леммы вытекает (3.1.6).

Лемма 3.4.3. $\Omega(Y)$ содержится в $\mathscr{S}$.

ДокаЗАТЕльство. $\Omega(Y) \subset \mathscr{A}$, поскольку $\mathscr{A}$-притягиваюшее множество. Кроме того, множество $\Omega(Y)$ инвариантно относительно групшы $W_{t}, t \in \mathbb{R}$, в силу непрерывности $W_{t}$ в $\mathscr{E}_{F}$. Поэтому для каж дого $\bar{Y} \in \Omega(Y)$ существует $C^{2}$-кривая $t \mapsto \lambda(t) \in \mathbb{R}$, для которой $W_{t} \bar{Y}=S_{\lambda(t)}$. Это значит, что $S_{\lambda(t)}$ - решение системы (3.2.1). Но тогда $\dot{\lambda}(t)=0$, т.е. $\lambda(t) \equiv \lambda$ и $\bar{Y}=S_{\lambda} \in \mathscr{S}$.

ii) Аналогично (0.1.23) доказьвается, что $\Omega(Y) \subset \mathscr{S}^{h}$ при $h=\mathscr{H}\left(Y^{0}\right)$. Следовательно, $Y(t) \stackrel{\mathscr{E}_{F}}{\longrightarrow} \mathscr{S}^{h}$ ввиду (3.1.6). Однако $\mathscr{S}^{h}$ - конечное множество по предложению 3.1.2. Поэтому (3.1.7) вытекает из непрерьвности $Y(t)$. 


\section{$\S$ 3.5. Притяжение к компактному множеству}

Выведем лемму 3.4.2 из следуюшей леммы о "притяжении в среднем", которая будет доказана в ближайшем параграфе. Обозначим для $R>0$

$$
\rho_{R}(t)=\inf _{S \in \mathscr{A}}\|Y(t)-S\|_{R} \text { при } t \in \mathbb{R} .
$$

ЛЕмма 3.5.1. Для любого $R>0$

$$
\int_{0}^{\infty} \rho_{R}^{2}(t) d t<\infty
$$

Зафиксируем любую метрику $\rho(\cdot, \cdot)$ на $\mathscr{E}$, определяюшую топологию $\mathscr{E}_{F}$. Докажем (3.4.1) от противного: пусть существуют такие $\varepsilon>0$ и последовательность $t_{k} \rightarrow \infty$, что

$$
\rho\left(Y\left(t_{k}\right), \mathscr{A}\right) \geqslant \varepsilon \text { при всех } k=1,2, \ldots
$$

Покажем, что это невозможно, и это завершит доказательство леммы 3.4.2. Можно считать, что $t_{k}+1<t_{k+1}$ для каждого $k$. Тогда из (3.5.2) по теореме Фату получаем

$$
\int_{0}^{1} \sigma_{R}(\theta) d \theta<\infty, \quad \text { где } \sigma_{R}(\theta)=\sum_{1}^{\infty} \rho_{R}^{2}\left(t_{k}+\theta\right) .
$$

Следовательно, $\sigma_{R}(\theta)<\infty$ для каждого $\theta \in \Theta(R)$, где $\Theta(R) \subset[0,1]$, причем $\int_{\Theta(R)} d x=1$. Тогда для любого $R>0$

$$
\rho_{R}\left(t_{k}+\theta\right) \rightarrow 0 \text { при } k \rightarrow \infty \text { для } \theta \in \Theta=\bigcap_{R \in \mathbb{N}}^{\infty} \Theta(R) .
$$

Следовательно, $Y\left(t_{k}+\theta\right) \stackrel{\mathscr{E}_{F}}{\longrightarrow} \mathscr{A}$ при $k \rightarrow \infty$ для каждого $\theta \in \Theta \subset[0,1]$, причем $\int_{\Theta} d x=1$. Тогда для любого $\theta \in \Theta$ из компактности $\mathscr{A}$ в $\mathscr{E}_{F}$ вытекает, что для некоторой последовательности $k(\theta) \rightarrow \infty$

$$
Y\left(t_{k(\theta)}+\theta\right) \stackrel{\mathscr{E}_{F}}{\longrightarrow} \bar{Y}(\theta) \in \mathscr{A} \text { при } k(\theta) \rightarrow \infty \text { для } \theta \in \Theta .
$$

Поэтому из непрерьвности отображения $W_{-\theta}$ в $\mathscr{E}_{F}$ следует также, что

$$
Y\left(t_{k(\theta)}\right) \stackrel{\mathscr{E}_{F}}{\longrightarrow} W_{-\theta} \bar{Y}(\theta) \text { при } k(\theta) \rightarrow \infty \text { для } \theta \in \Theta .
$$

С другой стороны, в силу компактности $\mathscr{A}_{\text {в } \mathscr{E} F}$ сушествует такая последовательность $\theta_{j} \in \Theta$, что $\theta_{j} \rightarrow 0$ при $j \rightarrow \infty$ и

$$
\bar{Y}\left(\theta_{j}\right) \stackrel{\mathscr{E}_{F}}{\longrightarrow} Y^{*} \in \mathscr{A} \text { при } j \rightarrow \infty .
$$

Наконец, в силу равномерной липшицевости (3.2.4) отображений $W_{-\theta}$ при $\theta \in[0,1]$ и сходимости $W_{-\theta_{j}} Y^{*} \stackrel{\mathscr{E}_{F}}{\longrightarrow} Y^{*}$ при $j \rightarrow \infty$ получаем

$$
W_{-\theta_{j}} \bar{Y}\left(\theta_{j}\right) \stackrel{\mathscr{E}_{F}}{\longrightarrow} Y^{*} \text { при } j \rightarrow \infty .
$$

Однако эта сходимость вместе с (3.5.7) при $\theta=\theta_{j}$ противоречит (3.5.3). 


\section{§3.6. Притяжение в среднем}

Мы доказываем здесь лемму 3.5.1. Достаточно построить для достаточно больших $\bar{\alpha}, \bar{\beta}, \bar{T}>0$ функцию $S_{\lambda(t)} \in \mathscr{A}=\mathscr{A}_{\bar{\alpha} \bar{\beta}}$, определенную при $t \geqslant \bar{T}$, для которой при любом $R>0$

$$
\int_{\bar{T}}^{\infty}\left\|Y(t)-S_{\lambda(t)}\right\|_{R}^{2} d t<\infty
$$

Мы покажем, что можно выбрать $\lambda(t)=u(-a, n)$ для $n \leqslant t<n+1, n=0,1, \ldots$. Можно заменить полунорму $\|\cdot\|_{R}$ из (2.1.6) на эквивалентную полунорму с $|u(-a)|$ вместо $|u(0)|$. Тогда (3.6.1) при $R>a$ означает, что

$$
\begin{aligned}
\int_{\bar{T}}^{\infty}\left(\int_{|x|<a}\left(\left|u^{\prime}(x, t)-s_{\lambda(t)}^{\prime}(x)\right|^{2}+|\dot{u}(x, t)|^{2}\right) d x+|u(-a, t)-\lambda(t)|^{2}\right. \\
\left.+\int_{a<|x|<R}\left(\left|u^{\prime}(x, t)\right|^{2}+|\dot{u}(x, t)|^{2}\right) d x\right) d t<\infty
\end{aligned}
$$

3.6.1. Рассеяние энергии в бесконечность. Справедлива оценка

$$
\int_{0}^{\infty}\left(\left|\dot{y}_{-}(t)\right|^{2}+\left|z_{-}(t)\right|^{2}+\left|\dot{y}_{+}(t)\right|^{2}+\left|z_{+}(t)\right|^{2}\right) d t<\infty
$$

где $y_{ \pm}(t)=u( \pm a, t)$ и $z_{ \pm}(t)=u^{\prime}( \pm a, t)$. Это вытекает аналогично (2.6.15) из представления Даламбера

$$
u(x, t)=f_{ \pm}(t-x)+g_{ \pm}(t+x), \quad \pm x>a, \quad t \in \mathbb{R}
$$

и конечности потока энергии в бесконечность, с использованием функционала энергии на отрезке $\Delta=[-a, a]:$ для $Y=(u(x), v(x)) \in \mathscr{E}$

$$
\mathscr{H}_{\Delta}(Y)=\int_{\Delta}\left[\frac{|v(x)|^{2}}{2}+\frac{\left|u^{\prime}(x)\right|^{2}}{2}+V(x, u(x))\right] d x .
$$

3.6.2. Нелинейная задача Гурса. Рассмотрим задачу Гурса для волнового уравнения (3.1.1) с данными Коши на прямых $x=$ const:

$$
\left\{\begin{array}{l}
\ddot{u}(x, t)=u^{\prime \prime}(x, t)+f(x, u(x, t)), \\
\left.u\right|_{x=r}=y(t),\left.\quad u^{\prime}\right|_{x=r}=z(t), \quad t \in \mathbb{R} .
\end{array}\right.
$$

Установим непрерьвность отображения $G_{r, x}:(y(\cdot), z(\cdot)) \mapsto\left(u(x, \cdot), u^{\prime}(x, \cdot)\right)$ и затем, используя эту непрерьвность, вьведем (3.6.2) из (3.6.3) в следуюшем разделе. 
ЗАмЕчАниЕ. Наши предположения (0.1.19), (0.1.20) обеспечивают глобальную корректность по $t$ задачи Коши (3.1.1), (3.1.2), но задача Гурса (3.6.6), вообше говоря, не является глобально корректной по $x \in \mathbb{R}$. Однако задача Гурса корректна локально по $x$, и этого достаточно для наших целей. Чтобы вывести (3.6.1) из (3.6.3), непрерьвность отображения $G_{b, x}$ необходимо доказать при $b=-a$ только для ограниченных $x \in[-R, R]$. Эта непрерьвность имеет место “для больших $t$ " и “вдоль" рассматриваемого (глобального) решения $u(x, t)$.

Пусть $\sigma$ обозначает произвольный отрезок длины $|\sigma|$ на $\mathbb{R}$.

ОПРЕДЕЛЕНИЕ 3.6.1. $\mathscr{E}(\sigma)$ - гильбертово пространство функций $(y(t), z(t)) \in$ $H^{1}(\sigma) \oplus L^{2}(\sigma)$, для которых

$$
\|(y, z)\|_{\mathscr{E}(\sigma)}=|\dot{y}|_{\sigma}+\|y\|_{\sigma}+|z|_{\sigma}<\infty,
$$

где $|\cdot|_{\sigma}$ - норма в $L^{2}(\sigma)$.

ОПРЕДЕлЕнИЕ 3.6.2. $\overline{\mathscr{E}}-$ пространство функций $(y(t), z(t)) \in H_{\mathrm{loc}}^{2}(\mathbb{R}) \oplus L^{2}(\mathbb{R})$, для которых

$$
\|(y, z)\|_{\overline{\mathscr{E}}}=\sup _{|\sigma| \geqslant 1} \frac{\|(y, z)\|_{\mathscr{E}(\sigma)}}{\sqrt{|\sigma|}}<\infty .
$$

ЗАмЕчАНИЕ. Из предложения 3.2.1 iii) и іv) следует, что $\left.\left(u, u^{\prime}\right)\right|_{x=r} \in \overline{\mathscr{E}}$ для каждого $r \in \mathbb{R}$ и, кроме того, $\left\|\left.\left(u, u^{\prime}\right)\right|_{x=r}\right\|_{\overline{\mathscr{E}}} \leqslant 2 e(r)$.

Рассмотрим решения $u(x, t)$ задачи Гурса (3.6.6) с $(y, z) \in \overline{\mathscr{E}}$, для которых $\left(u, u^{\prime}\right) \in$ $C(r-\varepsilon, r+\varepsilon ; \overline{\mathscr{E}})$ при некотором $\varepsilon>0$. Для таких решений задача Гурса эквивалентна интегральному равенству, аналогичному (3.2.1):

$$
Z(x)=W_{x-r}^{0} Z_{r}+\int_{r}^{x} W_{x-s}^{0}(0, f(s, u(s, \cdot))) d s,
$$

где $Z(x)=\left(u(x, \cdot), u^{\prime}(x, \cdot)\right)$ и $Z_{r}=(y(\cdot), z(\cdot))$.

Лемма 3.6.3. Пусть выполняются условия (0.1.18)-(0.1.20) и $Z_{r} \in \overline{\mathscr{E}}$. Тогда справедливы следующие утверждения.

i) Задача Гурса (3.66.) имеет единственное решение $Z(x)=: G_{r, x} Z_{r} \in C(r-\varepsilon$, $r+\varepsilon ; \overline{\mathscr{E}})$ c некоторым $\varepsilon>0$.

ii) $\varepsilon=\varepsilon(R, B)>0$ в i) зависит только от $R$ и В при $r \leqslant R u\left\|Z_{r}\right\|_{\overline{\mathscr{E}}} \leqslant B$.

iii) Для любих $R, B>0,|r| \leqslant R,\left\|Z_{r}\right\|_{\bar{E}} \leqslant B,|x-r|<\varepsilon(R, B)$ и каждого отрезка $\sigma \subset \mathbb{R}$ функиия $\left.Z(x, \cdot)\right|_{\sigma}$ зависит только от $\left.Z_{r}\right|_{\Sigma}$, әде $\Sigma$ - $\delta$-окрестность отрезка $\sigma$ в $\mathbb{R}, \delta=|x-r|$.

iv) Отображение $G_{r, x}:\left.\left.Z_{r}\right|_{\Sigma} \mapsto Z(x, \cdot)\right|_{\sigma}$ для $\left\|Z_{r}\right\|_{\overline{\mathscr{E}}} \leqslant B$ является непрерывныцм по Липшицу $\mathscr{E}(\Sigma) \rightarrow \mathscr{E}(\sigma):$

$$
\left\|G_{r, x} Z_{r}^{1}-G_{r, x} Z_{r}^{2}\right\|_{\mathscr{E}(\sigma)} \leqslant L(R, B)\left\|Z_{r}^{1}-Z_{r}^{2}\right\|_{\mathscr{E}(\Sigma)}
$$

при $|r| \leqslant R$ и $\delta=|x-r| \leqslant \varepsilon(R, B)$ для любых $Z_{r}^{j} \in \overline{\mathscr{E}}, j=1,2$. Константа Липчица $L(R, B)$ не зависит от отрезка $\sigma$. 
ДокАЗАТЕльство. Из принщипа сжимающих отображений вытекает существование и единственность такого решения $Z(x)$ задачи (3.6.9), что $Z(x) \in C(r-\varepsilon$, $r+\varepsilon ; \mathscr{E}(\sigma))$ для любого отрезка $\sigma \subset \mathbb{R}$. Решаюшее значение здесь имеет тот факт, что $\varepsilon=\varepsilon(R, B)>0$ не зависит от расположения отрезка $\sigma$ в силу равномерных оценок для $\left\|Z_{r}\right\|_{\mathscr{E}(\sigma)}$ при ограниченных $|\sigma| \geqslant 1$ и однородности задачи по $t$.

Свойства iii) и iv) вытекают из таких же свойств последовательных пикаровских приближений ввиду соответствующих свойств операторов $W_{x-s}^{0}$.

3.6.3. Доказательство притяжения в среднем. Вьведем (3.6.2) из (3.6.3) при помощи оценок (3.6.10). Мы полагаем $\lambda(t)=y_{-}(n) \equiv u(-a, n)$ для $n \leqslant t<n+1$, $n=0,1, \ldots$.

Шаг 1. Из оценок (3.6.3) и представления Даламбера (3.6.4) вытекает сходимость интеграла $\int_{\bar{T}}^{\infty} \int_{a<|x|<R} \cdots$ в (3.6.2).

Шаг 2. Интеграл $\int_{0}^{\infty}|u(-a, t)-\lambda(t)|^{2} d t$ также сходится, так как он равен

$$
\sum_{n=0}^{\infty} \int_{n}^{n+1}\left|y_{-}(t)-y_{-}(n)\right|^{2} d t \leqslant \int_{0}^{\infty}\left|\dot{y}_{-}(t)\right|^{2} d t<\infty
$$

Шаг 3. Проверим конечность ряда

$$
\sum_{n=\bar{N}}^{\infty} \int_{n}^{n+1}\left(\int_{-a}^{a}\left(\left|u^{\prime}(x, t)-s_{\lambda(n)}^{\prime}(x)\right|^{2}+|\dot{u}(x, t)|^{2}\right) d x\right) d t<\infty
$$

для достаточно больших $\bar{N}$. Предложение $3.2 .1 \mathrm{iv)} \mathrm{означает,} \mathrm{что} \mathrm{для} \mathrm{решения} Z(x)=$ $\left(u(x, \cdot), u^{\prime}(x, \cdot)\right)=G_{-a, x}\left(y_{-}(\cdot), z_{-}(\cdot)\right)$ уравнения $(3.6 .9)$

$$
\|Z(r)\|_{\overline{\mathscr{E}}} \leqslant \bar{B}=2 e(a) \text { при } r \in[-a, a] .
$$

С другой стороны, для любого $n=0,1, \ldots$ функция

$$
S_{n}(x)=\left(s_{\lambda(n)}(x), 0\right)=G_{-a, x}\left(y_{-}(n), 0\right)
$$

также является решением уравнения (3.6.9). Поэтому можно применить лишшищеву непрерьвность из леммы 3.6.3 iv), чтобы оценить разность меж ду этими двумя решениями.

Лемма 3.6.4. При всех достаточно больиих $n \geqslant \bar{N}$ существуют решения $S_{n}(x)=G_{-a, x}\left(y_{-}(n), 0\right)$ уравнения (3.6.9) и при каждом $x=-a+\delta \in[-a, a]$

$$
\left\|Z(x)-S_{n}(x)\right\|_{\mathscr{E}([n, n+1])}^{2} \leqslant \bar{L} \int_{n-\delta}^{n+1+\delta}\left(\left|z_{-}(t)\right|^{2}+\left|\dot{y}_{-}(t)\right|^{2}\right) d t \quad \partial \Omega_{\text {Я }} \quad n \geqslant \bar{N}
$$

Мы докажем эту лемму ниже. Суммируя (3.6.13) по $n \geqslant \bar{N}$ и интегрируя по $x \in$ $[-a, a]$, мы получаем (3.6.11) ввиду (3.6.3). 
Шаг 4. $S_{n}(x) \in \mathscr{A}_{\bar{\alpha} \bar{\beta}}$ при достаточно больших $\bar{\alpha}, \bar{\beta}>0$. Действительно, из (3.6.11) и оценки (3.2.7) следует, что для достаточно больших $\bar{N}$

$$
\bar{D}=\sup _{n \geqslant \bar{N}} \int\left|s_{\lambda(n)}^{\prime}(x)\right|^{2} d x<\infty
$$

Кроме того, из (3.2.5) при $x=-a$ вытекает

$$
\bar{d}=\sup _{n \geqslant 0}\left|s_{\lambda(n)}(-a)\right|<\infty
$$

Поэтому из (3.6.14) получаем аналогично (3.2.7)

$$
\sup _{n \geqslant 0}\left|s_{\lambda(n)}(x)\right| \leqslant \bar{\alpha}+\bar{\beta} \sqrt{x} \text { при }|x| \leqslant a,
$$

если $\bar{\alpha}$ и $\bar{\beta}$ достаточно велики.

ДОкАЗАТЕЛЬСТво ЛЕммЫ 3.6.4. Обозначим $\bar{\varepsilon}=\varepsilon(a, \bar{B})$ и докажем существование решения $S_{n}(x)=G_{-a, x}\left(y_{-}(n), 0\right)$ и оценки $(3.6 .13)$ при $-a+(k-1) \bar{\varepsilon} \leqslant x \leqslant-a+k \bar{\varepsilon}$ индукцией по $k=1,2, \ldots$ для $k \leqslant 2 a / \bar{\varepsilon}+1$.

$k=1$. На отрезке $-a \leqslant x \leqslant-a+\bar{\varepsilon}$ существование решения $S_{n}(x)=G_{-a, x}\left(y_{-}(n), 0\right)$ и оценки (3.6.13) для всех $n \geqslant 0$ непосредственно вытекают из (3.6.10) с $r=-a$ для двух решений $Z(x)$ и $S_{n}(x)$, так как $Z(-a)-S_{n}(-a)=\left(y_{-}(\cdot)-y_{-}(n), z_{-}(\cdot)\right)$ и

$$
\left\|Z(-a)-S_{n}(-a)\right\|_{\mathscr{E}([n-\delta, n+1+\delta])}^{2} \leqslant C(\delta) \int_{n-\delta}^{n+1+\delta}\left(\left|z_{-}(t)\right|^{2}+\left|\dot{y}_{-}(t)\right|^{2}\right) d t
$$

Оценка (3.6.10) применима к этим решениям в силу (3.6.12) с $r=-a$ и аналогичной оценки для $S_{n}(-a)=\left(y_{-}(n), 0\right)$.

$k=2$. На отрезке $-a+\bar{\varepsilon} \leqslant x \leqslant-a+2 \bar{\varepsilon}$ сушествование решения $S_{n}(x)=$ $G_{-a, x}\left(y_{-}(n), 0\right)=G_{-a+\bar{\varepsilon}, x} S_{n}(-a+\bar{\varepsilon})$ и оценки (3.6.13) получаются повторным применением (3.6.10) при достаточно больших $n \geqslant N_{1}$, для которых

$$
\left\|S_{n}(-a+\bar{\varepsilon})\right\|_{\overline{\mathscr{E}}} \leqslant \bar{B} \text { при } n \geqslant N_{1} .
$$

Такое $N_{1}<\infty$ сушествует в силу (3.12) и оценки (3.13) с $x=-a+\bar{\varepsilon}$, доказанной вьше, поскольку $\int_{n-\delta}^{n+1+\delta}\left(\left|z_{-}(t)\right|^{2}+\left|\dot{y}_{-}(t)\right|^{2}\right) d t \rightarrow 0$ при $n \rightarrow \infty$ в силу (3.6.3).

Индукция по $k$ завершает доказательство. 


\section{СПИСОК ЛИТЕРАТУРЫ}

[1] Бабин А. В., Вишик М. И. Аттракторы эволюционных уравнений. М. Наука, 1989.

[2] Balabane M., Cazenave T., Douady A., Merle F. Existence of excited states for a nonlinear Dirac field // Comm. Math. Phys. 1988. V. 119. P. 153-176.

[3] Berestycki H., Lions P. L. Nonlinear scalar field equations I; II // Arch. Rational Mech. Anal. 1983. V. 82. P. 313-345; 347-375.

[4] Bohr N. On the constitution of atoms and molecules // Phil. Mag. 1913. V. 26. P. 1-25.

[5] Buslaev V.S., Perel'man G.S. On nonlinear scattering of states which are close to a soliton // Astérisque. 1992. V. 208. P. 49-63.

[6] Buslaev V.S., Perel'man G.S. Scattering for the nonlinear Schrödinger equation: states close to a soliton // St. Petersburg Math. J. 1993. V. 4. №6. P. 1111-1142.

[7] Buslaev V.S., Perel'man G.S. On the stability of solitary waves for nonlinear Schrödinger equations // Amer. Math. Soc. Trans. Ser. 2. 1995. V. 164. P. 75-98.

[8] Chadam J. M. Asymptotics for $\square u=m^{2} u+G\left(x, t, u, u_{x}, u_{t}\right)$ I; II // Ann. Scuola Norm. Sup. Pisa Sci. Fis. Mat. (3). 1972. V. 26. P. 33-65; 67-95.

[9] Chadam J. M. On certain global solutions of the Cauchy problem for the (classical) coupled Klein-Gordon-Dirac equation in one and three space dimensions // Arch. Rational Mech. Anal. 1974. V. 54. P. 223-237.

[10] Егоров Ю. Е. О стабилизации взаимодействия струны с нелинейными осцилляторами // Дис.... канд. физ.-матем. наук. М.: МГУ, 1997.

[11] Егоров Ю. Е., Комеч А. И. Об аттракторах и асимптотике решений в нелинейной задаче Лэмба // Вестник МГУ. Сер. 1. Матем., мех. 1996. № 5. С. 80-88.

[12] Егоров Ю. Е., Комеч А. И. Стабилизация взаимодействия струны с нелинейным осциллятором // УМН. 1995. V. 50. № 4. Р. 152.

[13] Esteban M. J., Georgiev V., Séré E. Stationary solutions of the Maxwell-Dirac and Klein-Gordon-Dirac equations // Calc. Var. Partial Differential Equations. 1996. V. 4. № 3. P. 265-281.

[14] Esteban M. J., Séré E. Stationary states of the nonlinear Dirac equation: a variational approach // Comm. Math. Phys. 1995. V. 171. № 2. P. 323-350.

[15] Фейнман Р., Лейтон Р., Сэндс М. Фейнмановские лекции по физике. Электродинамика. М.: Мир, 1966.

[16] Gell-Mann M. Symmetries of baryons and mesons // Phys. Rev. 1962. V. 125. P. 1067-1084.

[17] Ginibre J., Velo G. Time decay of finite energy solutions of the nonlinear Klein-Gordon and Schrödinger equations // Ann. Inst. H. Poincaré Phys. Théor. 1985. V. 43. P. 399-442.

[18] Glassey R. T., Strauss W. A. Decay of classical Yang-Mills fields // Comm. Math. Phys. 1979. V. 65. P. 1-13.

[19] Glassey R.T., Strauss W.A. Decay of Yang-Mills field coupled to a scalar field // Comm. Math. Phys. 1979. V. 67. P. 51-67.

[20] Grillakis M., Shatah J., Strauss W. A. Stability theory of solitary waves in the presence of symmetry, I; II // J. Funct. Anal. 1987. V. 74. P. 160-197; 1990. V. 94. P. 308-348.

[21] Hale J. Asymptotic Behavior of Dissipative Systems. Providence: Amer. Math. Soc., 1988.

[22] Hörmander L. On the fully nonlinear Cauchy problem with small data // IMA Vol. Math. Appl. 1991. V. 30. P. 51-81.

[23] Джексон Д. Классическая электродинамика. М.: Мир, 1965.

[24] Keller J. B., Bonilla L. L. Irreversibility and nonrecurrence // J. Statist. Phys. 1986. V. 42. №5/6. P. 1115-1125.

[25] Хруслов Е.Я. Асимптотика решения задачи Коши для уравнения Кортевега-де Фриза с начальными данными типа ступеньки // Матем. сб. 1976. Т. 99. № 2. С. 261-281.

[26] Klainerman S. Long-time behavior of solutions to nonlinear evolution equations // Arch. Rational Mech. Anal. 1982. V. 78. P. 73-98. 
[27] Комеч А. И. О стабилизации взаимодействия струны с нелинейным осциллятором // УМН. 1991. Т. 46. №6. С. 166-167.

[28] Комеч А. И. Стабилизация взаимодействия струны с нелинейным осциллятором // Вестник МГУ. Сер. 1. Матем., мех. 1991. №6. С. 35-41.

[29] Komech A. I. On stabilization of string-nonlinear oscillator interaction // J. Math. Anal. Appl. 1995. V. 196. P. 384-409.

[30] Комеч А. И. О переходах к стационарным состояниям в некоторых бесконечномерных гамильтоновых системах // Докл. РАН. 1996. Т. 347. № 3. С. 309-311.

[31] Комеч А. И. О стабилизации взаимодействия струны с конечным числом осцилляторов // УМH. 1994. Т. 49. № 4. С. 117.

[32] Komech A. I. On the stabilization of string-oscillator interaction // Russian J. Math. Phys. 1995. V. 3. P. 227-247.

[33] Komech A. I. On transitions to stationary states in one-dimensional nonlinear wave equations // Arch. Rational Mech. Anal. 1999. V. 149. № 3. P. 213-228.

[34] Komech A. I. On transitions to stationary states in Hamiltonian nonlinear wave equations // Phys. Lett. A. 1998. V. 241. P. 311-323.

[35] Komech A. I., Kunze M., Spohn H. Effective dynamics for a mechanical particle coupled to a wave field // Comm. Math. Phys. 1999. V. 203. P. 1-19.

[36] Komech A. I., Spohn H. Soliton-like asymptotics for a classical particle interacting with a scalar wave field // Nonlinear Anal. 1998. V. 33. P. 13-24.

[37] Komech A. I., Spohn H. Long-time asymptotics for the coupled Maxwell-Lorentz equations // Comm. Partial Differential Equations. (to appear).

[38] Komech A. I., Spohn H., Kunze M. Long-time asymptotics for a classical particle interacting with a scalar wave field // Comm. Partial Differential Equations. 1997. V. 22. № 1/2. P. 307-335.

[39] Komech A. I., Vainberg B. R. On asymptotic stability of stationary solutions to nonlinear wave and Klein-Gordon equations // Arch. Rational Mech. Anal. 1996. V. 134. P. 227-248.

[40] Ладыженская О. А. Об аттракторах нелинейных эволюционных диссипативных задач // Зап. науч. семин. ЛОМИ. 1986. Т. 18. С. $72-85$.

[41] Lamb H. On a peculiarity of the wave-system due to the free vibrations of a nucleus in an extended medium // Proc. London Math. Soc. 1900. V. 32. P. 208-211.

[42] Lax P.D., Morawetz C.S., Phillips R.S. Exponential decay of solutions of the wave equation in the exterior of a star-shaped obstacle // Comm. Pure Appl. Math. 1963. V. 16. P. 477-486.

[43] Лакс П., Филлипс Р. Теория рассеяния. М.: Мир, 1971.

[44] Morawetz C.S. The decay of solutions to exterior initial-boundary value problem for the wave equation // Comm. Pure Appl. Math. 1961. V. 14. P. 561-568.

[45] Morawetz C.S., Strauss W. A. Decay and scattering of solutions of a nonlinear relativistic wave equation // Comm. Pure Appl. Math. 1972. V. 25. P. 1-31.

[46] Ne'emann Y. Derivation of strong interactions from a gauge invariance // Nuclear Phys. 1961. V. 26. P. 222-229.

[47] Новокшенов В. Ю. Асимптотика при $t \rightarrow \infty$ решения задачи Коши для нелинейного уравнения Шрёдингера // Докл. АН СССР. 1980. Т. 251. № 4. С. 799-801.

[48] Pillet C.-A., Wayne C.E. Invariant manifolds for a class of dispersive, Hamiltonian, partial differential equations // J. Differential Equations. 1997. V. 141. № 2. P. 310-326.

[49] Reed M. Abstract non-linear wave equations. Berlin: Springer-Verlag, 1976. (Lecture Notes in Math. V. 507.)

[50] Roffman E. H. Localized solutions of nonlinear wave equations // Bull. Amer. Math. Soc. 1970. V. 76. P. $70-71$. 
[51] Сазонов Л. И., Юдович В. И. Устойчивость стационарных решений параболических уравнений и уравнения Навье-Стокса во всем пространстве // Сиб. матем. журн. 1988. T. 29. №1. C. 151-158.

[52] Шрёдингер Э. Что такое жизнь? М.: ИЛ, 1947.

[53] Segal I. Dispersion for nonlinear relativistic wave equations // Ann. Sci. Ecole Norm. Sup. 1968. V. 1. P. 459-497.

[54] Шабат А. Б. Об уравнениях Кортевега-де Фриса // Докл. АН СССР. 1973. Т. 211. № 6. C. 1310-1313.

[55] Soffer A., Weinstein M. I. Multichannel nonlinear scattering for nonintegrable equations // Comm. Math. Phys. 1990. V. 133. P. 119-146.

[56] Soffer A., Weinstein M. I. Multichannel nonlinear scattering for nonintegrable equations. II. The case of anisotropic potentials and data // J. Differential Equations. 1992. V. 98. № 2. P. 376-390.

[57] Strauss W. A. Decay and asymptotics for $\square u=F(u) / /$ J. Funct. Anal. 1968. V. 2. P. $409-457$.

[58] Strauss W. A. Nonlinear Invariant Wave Equations. Berlin: Springer-Verlag, 1978. (Lecture Notes in Phys. V. 73.)

[59] Суханов В.В. Асимптотическое поведение решений задачи Коши для системы типа КдВ при больших временах // Докл. АН СССР. 1983. Т. 269. №5. С. 1091-1094.

[60] Temam R. Infinite-Dimensional Dynamical Systems in Mechanics and Physics. Berlin: Springer-Verlag, 1988. (Appl. Math. Sci. V. 68.)

[61] Вайнберг Б. Р. Об аналитических свойствах резольвенты для некоторого класса пучков операторов // Матем. сб. 1968. Т. 77. С. 259-296.

[62] Вайнберг Б. Р. Поведение решений задачи Коши для гиперболических уравнений при $t \rightarrow \infty / /$ Матем. сб. 1969. Т. 78. №4. С. 542-578.

[63] Вайнберг Б.Р. Асимптотическое поведение при $t \rightarrow \infty$ решений внешних смешанных задач для гиперболических уравнений и квазиклассика // Итоги науки и техники. Современные проблемы математики. Фундаментальные направления. Т. 34. М.: ВИНИТИ, 1991. C. 57-92.

[64] Вайнберг Б.Р. Асимптотические методы в уравнениях математической физики. М.: Изд-во МГУ, 1982.

[65] Weinberg S. Conservation of isotopic spin and isotopic gauge invariant // Rev. Modern Phys. 1980. V. 52. P. 515.

[66] Wheeler J. A., Feynman R. P. Interaction with the absorber as the mechanism of radiation // Rev. Modern Phys. 1945. V. 17. P. 157-181.

[67] Yang C. N., Mills R. L. // Phys. Rev. 1954. V. 96. № 1. P. 191-195.

Московский государственный

Поступила в редакцию университет им. М. В. Ломоносова 19.08.1998

E-mail: komech@sci.lpi.ac.ru 